\title{
Chromosome-level genome assemblies of five Prunus species and genome-wide association studies for key agronomic traits in peach
}

Qiuping Tan ${ }^{1,2,3,4}$, Sen Li $i^{2,3,4}$, Yuzheng Zhang ${ }^{2,3,4}$, Min Chen ${ }^{5}$, Binbin Wen ${ }^{2,3,4}$, Shan Jiang ${ }^{2,3,4}$, Xiude Chen ${ }^{2,3,4}$, Xiling Fu $\mathrm{Fu}^{2,3,4}$, Dongmei $\mathrm{Li}^{2,3,4}$, Hongyu Wu $\mathrm{W}^{2,6}$, Yong Wang ${ }^{1,2}$, Wei Xiao ${ }^{2,3,4 凶}$ and Ling $\mathrm{Li}^{2,3,4 凶}$

\begin{abstract}
Prunus species include many important perennial fruit crops, such as peach, plum, apricot, and related wild species. Here, we report de novo genome assemblies for five species, including the cultivated species peach (Prunus persica), plum (Prunus salicina), and apricot (Prunus armeniaca), and the wild peach species Tibetan peach (Prunus mira) and Chinese wild peach (Prunus davidiana). The genomes ranged from 240 to $276 \mathrm{Mb}$ in size, with contig N50 values of 2.27-8.30 Mb and 25,333-27,826 protein-coding gene models. As the phylogenetic tree shows, plum diverged from its common ancestor with peach, wild peach species, and apricot $\sim 7$ million years ago (MYA). We analyzed wholegenome resequencing data of 417 peach accessions, called 3,749,618 high-quality SNPs, 577,154 small indels, 31,800 deletions, duplications, and inversions, and 32,338 insertions, and performed a structural variant-based genome-wide association study (GWAS) of key agricultural traits. From our GWAS data, we identified a locus associated with a fruit shape corresponding to the OVATE transcription factor, where a large inversion event correlates with higher OVATE expression in flat-shaped accessions. Furthermore, a GWAS revealed a NAC transcription factor associated with fruit developmental timing that is linked to a tandem repeat variant and elevated NAC expression in early-ripening accessions. We also identified a locus encoding microRNA172d, where insertion of a transposable element into its promoter was found in double-flower accessions. Thus, our efforts have suggested roles for OVATE, a NAC transcription factor, and microRNA172d in fruit shape, fruit development period, and floral morphology, respectively, that can be connected to traits in other crops, thereby demonstrating the importance of parallel evolution in the diversification of several commercially important domesticated species. In general, these genomic resources will facilitate functional genomics, evolutionary research, and agronomic improvement of these five and other Prunus species. We believe that structural variant-based GWASs can also be used in other plants, animal species, and humans and be combined with deep sequencing GWASs to precisely identify candidate genes and genetic architecture components.
\end{abstract}

\section{Introduction}

The Rosaceae family includes many genera with different types of fruit, of which the Prunus genus contains

\footnotetext{
Correspondence: Wei Xiao (gulight986918@163.com) or

Ling Li (lilingsdau@163.com)

${ }^{1}$ College of Life Sciences, Shandong Agricultural University, Tai'an 271018 People's Republic of China

${ }^{2}$ State Key Laboratory of Crop Biology, Shandong Agricultural University, Tai'an 271018, People's Republic of China

Full list of author information is available at the end of the article
}

several fruit tree species with important economic value, such as peach (Prunus persica), plum (Prunus salicina), and apricot (Prunus armeniaca), as well as wild peach species such as Tibetan peach (Prunus mira) and Chinese wild peach (Prunus davidiana). The fleshy fruit of Prunus crops offers an abundance of nutrients, such as carbohydrates, organic acids, vitamins, and minerals. Extensive phenotypic variation contributing to these fruit characteristics exists within or between species. Although these

\section{(c) The Author(s) 2021}

\footnotetext{
(cc) Open Access This article is licensed under a Creative Commons Attribution 4.0 International License, which permits use, sharing, adaptation, distribution and reproduction cc in any medium or format, as long as you give appropriate credit to the original author(s) and the source, provide a link to the Creative Commons license, and indicate if changes were made. The images or other third party material in this article are included in the article's Creative Commons license, unless indicated otherwise in a credit line to the material. If material is not included in the article's Creative Commons license and your intended use is not permitted by statutory regulation or exceeds the permitted use, you will need to obtain permission directly from the copyright holder. To view a copy of this license, visit http://creativecommons.org/licenses/by/4.0/.
} 
species have considerable economic value, the genetic mechanisms underlying favorable traits are poorly understood. This lack of understanding is, to some extent, attributed to the scarcity of genomic resources, which severely limits efforts to improve Prunus crops, particularly for plum, Prunus mira, and Prunus davidiana. Owing to their small genome sizes $(\sim 250 \mathrm{Mb})$ and relatively short juvenile periods ( $2-3$ years), most of the aforementioned Prunus species are promising candidates for functional and evolutionary studies of the Rosaceae family, particularly peach ${ }^{1}$, which originated in China, was domesticated 4,000 years ago and was subsequently dispersed to Europe through the Silk Road ${ }^{2}$. Extensive genetic efforts have identified various quantitative trait loci (QTLs) that influence many important traits in peach ${ }^{3}$, but only a few genes have been identified as candidate genes for traits such as flesh texture and adhesion ${ }^{4}$, flesh color $^{5}$, fruit hairlessness ${ }^{6}$, dormancy ${ }^{7}$, and tree architecture ${ }^{8}$.

Genome-wide association studies (GWASs) have identified many candidate genes for key traits in Arabidopsis $^{9}$, rice ${ }^{10}$, maize ${ }^{11}$, tomato ${ }^{12}$, and upland cot$\operatorname{ton}^{13}$. The detection power of GWASs is majorly affected by two factors ${ }^{14}$. The first is population structure, and the second is linkage disequilibrium (LD), which is species-specific and genomic interval-specific and determines the resolution of the GWAS. The single nucleotide polymorphism (SNP)-based GWAS approach uses LD to relate the top associated SNPs to flanking genes and directly or indirectly identifies candidate genes and genetic architecture components ${ }^{15}$. However, in some cases, the SNPs with the strongest associations may be very far from the candidate gene because of the high LD in the candidate interval ${ }^{9,10}$. When LD is low in some species, such as peach, candidate genes may not be identified due to nonlinkage of the strongest associated SNPs with these genes ${ }^{16,17}$. Furthermore, SNP-based GWASs easily identify the biological effects of most associated SNPs on a phenotype when the SNP is located within regions of the gene body or the flanking interval ${ }^{10,11}$; however, it cannot directly do so when the SNP is located within the intergenic interval. Unfortunately, this so-called "junk DNA" interval often comprises the majority of the genome, such as $\sim 97 \%$ in the human genome ${ }^{18}$. Very few intergenic SNPs have a significant effect on phenotype; however, structural variants (SVs) located at intergenic regions have been found to have major effects on traits ${ }^{19-21}$. Furthermore, the candidate genetic architecture components for the phenotype will have a stronger associated signal than that of its flanking SNP in GWASs, as crossover and recombination reduce the linkage of these noncausal SNPs with phenotypes. Other factors, such as genetic drift and mutation, may also affect the linkage of noncausal SNPs with phenotypes ${ }^{22}$.
Genetic variants rather than SNPs may be directly responsible for phenotypes $^{11}$, considering that the genetic architecture for traits is complex and diversified.

Large structural variations (SVs), defined as at least $50 \mathrm{bp}$ in size, were recently recognized as important variant types for traits and diseases in plants, animal species, and humans ${ }^{20,21,23}$. These SVs mainly include deletions (DELs), inversions (INVs), duplications (DUPs), insertions (INSs or LIs), and translocations (BNDs). Most traditional SNP-based GWASs are performed on samples with a relatively shallow depth of sequencing $(\sim 5 \mathrm{x})$; however, this can increase the uncertainty of genotyping and the missing rate. Occasionally, the candidate bases for a trait will be lost after filtering. Thus, deep sequencing of samples (20x, or even 30x) is recommended for SV-based GWASs. Although many tools for discovering SVs have been developed ${ }^{24-26}$, very few software tools can be used to call and genotype SVs at a large population scale. Thus, unlike classic SNP-based GWASs, GWASs based on SVs at a large population scale are rarely reported.

In this study, we report five de novo genome assemblies for peach, plum, apricot, and the wild peach species Prunus mira and Prunus davidiana. We additionally analyzed whole-genome resequencing data of 417 peach accessions and used an SV-based GWAS approach to explore the candidate genes and genetic architecture for key agronomic traits in peach. Using this approach, we successfully identified a number of new candidate genes and genetic architectures influencing key agronomic traits. These genomic resources represent a possible foundation for functional and evolutionary studies and will aid in marker-assisted breeding in Prunus species in the future. We believe that this approach can also be used in other plants, animal species, and humans combined with deep-sequencing GWASs to precisely identify candidate genes and genetic architecture components.

\section{Results \\ Genome assembly}

In this study, we de novo assembled the plum, Prunus mira, and Prunus davidiana genomes for the first time and improved the peach and apricot genomes by integrating single-molecule real-time (SMRT) long-read sequencing (PacBio), short high-quality Illumina paired-end sequencing, and $\mathrm{Hi}-\mathrm{C}$ technology. First, we used SMRT reads $(99-130 \mathrm{~Gb}, 396-520$-fold coverage of estimated genomes, Supplementary Table 1) to assemble contigs, and we captured $244-276 \mathrm{Mb}$ initial genome assemblies consisting of $122-315$ contigs with an N50 ranging from 2.27 to $8.30 \mathrm{Mb}$ (Supplementary Table 2). Second, the aforementioned SMRT reads and clean Illumina paired-end reads (55-59 G, 220-236fold coverage of estimated genomes, Supplementary Table 3) were used to correct and polish the initially 
assembled contigs. Third, the initially assembled contigs were categorized and ordered into pseudochromosomes using $\mathrm{Hi}-\mathrm{C}$ sequencing data $(25-42 \mathrm{G}, 100$ -168-fold coverage of estimated genomes, Supplementary Table 4). The resulting final genome size ranged from 240 to $267 \mathrm{Mb}, 94-99 \%$ of the genome was anchored to 8 chromosome-scale scaffolds, with the $\mathrm{N} 50$ of scaffolds ranging from 27.79 to $31.53 \mathrm{Mb}$ and with a gap number from 75 to 229 (Supplementary Table 5). Compared to a previously published Lovell peach assembly ${ }^{3}(227 \mathrm{Mb}$ of genome size), which consisted of 2,525 contigs with an N50 of $\sim 250 \mathrm{~kb}$ (https://www.rosaceae.org), our final peach assembly consisted of 315 contigs with an N50 of $\sim 4,640 \mathrm{~kb}$ and resulted in a reduction in the gap number from $\sim 2,300$ to $\sim 140$ in the final assembly. Therefore, our peach assembly showed an $\sim 18$-fold increase in the length of the contig N50 and 16-fold fewer gaps than the Lovell assembly. Compared with the recently released apricot assembly $^{27}$ ( $220 \mathrm{Mb}$ of genome size), which consisted of 444 contigs with an N50 of $1.02 \mathrm{Mb}$, our apricot assembly consisted of 122 contigs with an N50 of $3.31 \mathrm{Mb}$ and resulted in a reduction in the gap number from 241 to 163 . Our apricot assembly, therefore, showed an $\sim 3.25$-fold increase in the length of the contig N50 and $~ 1.48$-fold fewer gaps compared with the recently published apricot assembly. Our five assembled Prunus genomes depicted high congruence because the strongest signals from the $\mathrm{Hi}-\mathrm{C}$ data clustered at the expected diagonal (Fig. S1). Strong collinear relationships existed among Prunus genomes (Fig. S2), indicating that our pseudochromosomes derived from anchored and oriented contigs were of high quality. We also mapped the clean Illumina short DNA reads to their respective assemblies with a mapping ratio from 94 to $98 \%$, which further supported the accuracy and completeness of the genome assembly. These highquality genomes offer the opportunity to study the evolutionary relationships among genomes.

\section{Genome annotation}

Genome annotation was based on full-length RNA-iso sequencing and homology-based and de novo prediction strategies. We obtained $25,333-27,826$ protein-coding gene models in these five Prunus species (Supplementary Table 6). A total of $93.70-98.10 \%$ of the complete orthologs were detected in these assemblies based on the 1,375 Benchmarking Universal Single-Copy Orthologs (BUSCOs) $^{28}$ (Supplementary Table 7). Based on these complete assemblies, we predict that $43.30-50.13 \%$ of the genomes were composed of repeat sequences (Supplementary Table 8 ). The most highly present mobile elements in these species were of the "unknown" type (Supplementary Table 9).

\section{Phylogenetic analysis}

To investigate the evolutionary history of these Prunus species within Rosaceae, we constructed a maximum likelihood phylogenetic tree using these five Prunus species and six other representatives Rosaceae species, including apple (Malus domestica), woodland strawberry (Fragaria vesca), almond (Prunus dulcis), peach (Prunus persica), mei (Prunus mume), and sweet cherry (Prunus avium). From the results of gene family clustering, 248 single-copy orthologous genes were used for tree construction and species divergence time estimation. As the phylogenetic tree shows (Fig. S3), plum was placed as a sister species adjacent to apricot and mei. Furthermore, wild peach species were sister species with cultivars of peach, as expected. We estimated that plum diverged from the common ancestor shared with apricot and mei $\sim 5$ million years ago (MYA). According to our data, plum diverged from its common ancestor with peach, wild peach species, and apricot $\sim 7$ million years ago (MYA).

\section{Characterization of the $\mathbf{4 1 7}$ peach accessions}

In this study, we analyzed 417 worldwide peach accessions; the 159 publicly obtained accessions had different coverage depths $(3 x-100 x)$, and the remaining 258 accessions were newly sequenced in this study. Furthermore, 182 of these 258 accessions were sequenced twice and merged at $\sim 20 \mathrm{x}$ (each is $\sim 10 \mathrm{x}$ ), and the other 78 accessions were sequenced at $\sim 10 x$. We called a total of 3,749,618 high-quality SNPs and 577,154 small indels from the 417 accessions. Additionally, we identified 31,800 DELs, DUPs, and INVs and 32,338 LIs from 326 deeply sequenced cultivars. We used 99,265 pruned SNPs for the phylogenetic tree, population structure, PCA, and LD decay analyses of the 417 accessions. From the phylogenetic tree (Fig. 1a), we grouped the 417 accessions into three subpopulations, wild, landrace, and improved accessions, which was further supported by PCA, which slightly separated most landraces and improved accessions (Fig. 1b), and a population structure plot (Fig. 1c). There was no clear population structure in the PCA plot when 18 wild accessions were excluded (Fig. 1b). With LD decay analyses, we found that wild accessions had the lowest $\mathrm{LD}$ value $\left(\sim 10 \mathrm{k} ; r^{2}=0.39\right)$, the improved accessions had the highest value $\left(\sim 50 \mathrm{k} ; r^{2}=0.39\right)$, and the LD values for the whole population were $\sim 25 \mathrm{k}$ at $r^{2}=0.39$. These values were comparable to those estimated in a previous study ${ }^{29}$ and in cultivated maize ${ }^{30}(22-30 \mathrm{~kb})$ but far smaller than those estimated in rice ${ }^{10}(\sim 167 \mathrm{~kb})$ and $\operatorname{cotton}^{31}(\sim 145.5 \mathrm{~kb})$. The characterization of this GWAS population suggested that this population was suitable for performing GWASs according to its small LD decay value and lack of population structure. Using this population, we identified a number of candidate gene loci for key agronomic traits in peach (Table 1). 


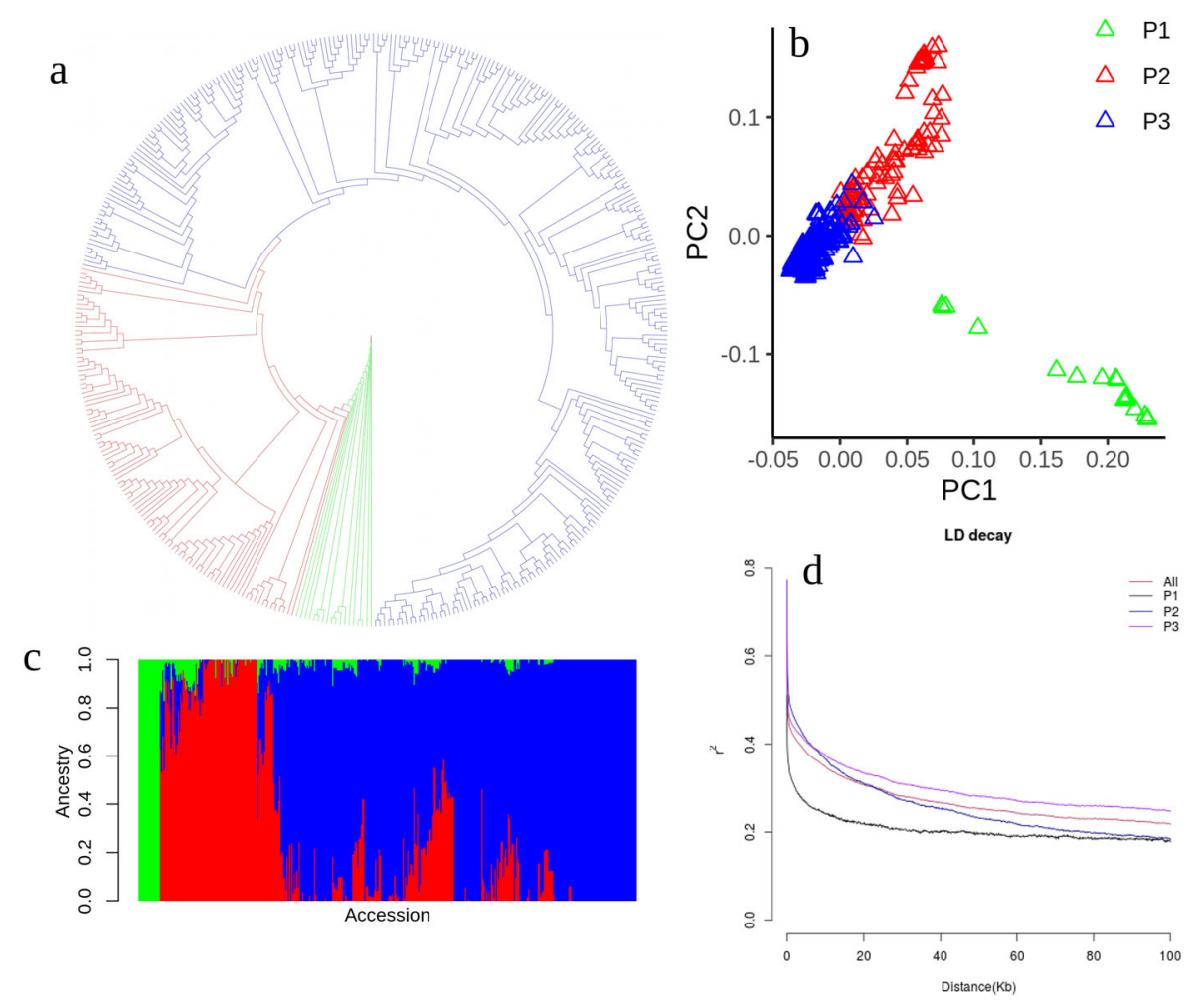

Fig. 1 Phylogenetic tree, PCA, population structure, and LD decay of the $\mathbf{4 1 7}$ peach accessions. a Neighbor-joining tree of this GWAS population based on a whole-genome filtered high-quality SNP dataset. b PCA plot of the first two components (PC1 and PC2). c Population structure with $K=3$. The $y$-axis represents the proportion of the ancestral relationship, and the $x$-axis indicates the accessions. The accessions on the $x$-axis were ordered in the same way as those in the phylogenetic tree. $\mathbf{d}$ Genome-wide average LD decay was estimated from four populations: All, P1, P2, and P3. The green color in $\mathbf{a}, \mathbf{b}$, and $\mathbf{c}$ stands for wild accessions; the red color in $\mathbf{a}, \mathbf{b}$, and $\mathbf{c}$ stands for landrace accessions; and the blue color in $\mathbf{a}, \mathbf{b}$, and $\mathbf{c}$ stands for improved accessions. In $\mathbf{d}$ all, P1, P2, and P3 stand for whole accessions, wild, landrace, and improved accessions, respectively

\section{GWAS of fruit shape}

Fruit shape in peach was generally classified into round and flat, and previous studies have shown that the flat shape was dominant to the round shape and regulated by a major gene mapped to the distal end of chromosome $6^{32-34}$. Previous GWASs have discovered that some SNPs in chromosome 6 are closely associated with this trait ${ }^{16,17}$. In this study, we first performed a SNP-based GWAS and identified a SNP with the strongest association (chr6:28,973,642; A/T; $P=1.17 \mathrm{e}-35$ ) (Fig. S4a). We later applied an SV-based GWAS approach to this trait. We identified a large inversion event (chr6:26,847,156; $P=$ $1.35 \mathrm{e}-84 ; \sim 1.67 \mathrm{Mb}$ in size) that showed the strongest association with this trait (Fig. 2a). The upstream end of this inversion event was located $\sim 3 \mathrm{~kb}$ downstream of Prupe.6G290900 (Fig. 2c), which encodes an ovate family protein (OFP) whose ortholog in tomato was a key fruit shape controlling gene $\mathrm{e}^{35}$. The downstream region of this inversion variant was located upstream of Prupe.6G323700, which encodes the activator subunit of the SNF1 complex; this gene regulates energy dynamic equilibrium in cells ${ }^{36}$. The upstream region of this inversion variant is $\sim 2.13 \mathrm{Mb}$ away from the top associated SNP identified using the SNP-based GWAS approach in this study and is $\sim 0.08 \mathrm{Mb}$ away from the top associated SNP identified by a previous study ${ }^{17}$. There were two main haplotypes in the SV-based GWAS panel based on this inversion variant (Fig. 2d). All accessions $(n=274)$ with Hap.1 (reference genome) had round-type fruit, while all accessions $(n=34)$ with Hap.2 had flat-type fruit. To validate whether this inversion event was responsible for the flat peach trait, we first analyzed the expression patterns of flanking genes during fruit development based on public RNA-seq data ${ }^{37}$. We found that Prupe.6G290900 expression was significantly higher in flat-type fruit than in round-type fruit at each time point (Fig. 2e), especially at 0 and 15 DAFB (Days After Full Bloom), which are critical stages for fruit shape determination. We further detected the expression of Prupe.6G290900 at 40 DAFB in 48 accessions. At the population level scale, the expression of Prupe.6G290900 in the flat-type population $(n=$ 23) was higher than that in the round-type population $(n=25)$ (Fig. 2f). At the individual level, in each flat-type fruit $(n=23)$, the expression of Prupe.6G290900 was 
Table 1 Trait-associated genetic markers and candidate genes from GWAS analysis

\begin{tabular}{|c|c|c|c|c|c|c|c|c|}
\hline Traits & QTL & Chromosome & Position & Marker & MAF & $-\log P$ & Gene & Annotation \\
\hline Fruit shape & qFS6 & 6 & $26,847,156$ & INV & 0.06 & 83.67 & Prupe.6G290900 & Ovate family protein 1-related \\
\hline Aborted fruit & qAF6 & 6 & $26,847,156$ & INV & 0.06 & 83.67 & Prupe.6G323700 & Sucrose nonfermenting 4-like protein \\
\hline Fruit sugar content & qFSC5 & 5 & 720,760 & $\mathrm{G} / \mathrm{T}$ & 0.32 & 2.28 & Prupe.5G006300 & $\begin{array}{l}\text { Tonoplast monosaccharide } \\
\text { transporter } 2\end{array}$ \\
\hline \multirow[t]{2}{*}{ Fruit development period } & qFDP4 & 4 & $11,127,010$ & DEL & 0.49 & 7.65 & Prupe.4G187100 & NAC domain-containing protein 2 \\
\hline & qFDP5 & 5 & $2,191,169$ & $C / G$ & 0.07 & 7.43 & Prupe.5G019900 & Ankyrin repeat family protein \\
\hline \multirow[t]{2}{*}{ Flower double } & qFD2 & 2 & $25,860,343$ & $\mathrm{LI}$ & NA & 82.00 & Prupe.2G237700 & MicoRNA172d \\
\hline & qFD6 & 6 & 21609914 & $A / G$ & 0.06 & 39.45 & Prupe.6G207400 & Elicitor-activated gene 3-2 \\
\hline \multirow[t]{2}{*}{ Flesh color } & $\mathrm{qFC1}$ & 1 & $26,614,905$ & $\mathrm{LI}$ & NA & 4.92 & Prupe.1G255500 & $\begin{array}{l}\text { Nine-cis-epoxycarotenoid } \\
\text { dioxygenase } 4\end{array}$ \\
\hline & $\mathrm{qFC} 4$ & 4 & $15,447,272$ & $A / G$ & 0.10 & 8.09 & Prupe.4G237000 & UDP-Glycosyltransferase superfamily \\
\hline Fruit hairiness & $\mathrm{qFH} 5$ & 5 & $15,893,169$ & $\mathrm{LI}$ & NA & 16.96 & Prupe.5G196100 & MYB domain protein 16 \\
\hline Flower morphology & qFM8 & 8 & $14,501,676$ & DEL & 0.31 & 16.47 & Prupe.8G118300 & Unknown protein \\
\hline Fruit nonacidity & qFNA5 & 5 & 628,841 & $\mathrm{~T} / \mathrm{TG}$ & 0.48 & 8.13 & Prupe.5G005400 & Switch subunit 3 \\
\hline Flesh adhesion & qFA8 & 8 & $18,818,363$ & G/GGTTAA & 0.21 & 8.31 & Prupe.8G196700 & Cyclin family protein \\
\hline Flesh texture & qFT4 & 4 & $10,270,803$ & $\mathrm{G} / \mathrm{A}$ & 0.07 & 8.76 & Prupe.4G173600 & UDP-Glycosyltransferase superfamily \\
\hline \multirow[t]{2}{*}{ Flesh color around stone } & qFCAS3 & 3 & $8,910,191$ & LI & NA & 7.55 & Prupe.3G109700 & Pectin lyase-like superfamily protein \\
\hline & qFCAS5 & 5 & $14,370,757$ & $\mathrm{TA} / \mathrm{T}$ & 0.28 & 6.92 & Prupe.5G165400 & Unknown protein \\
\hline \multirow[t]{2}{*}{ Male sterility } & qMS3 & 3 & $23,629,984$ & T/A & 0.10 & 8.41 & Prupe.3G243900 & S-adenosylmethionine decarboxylase \\
\hline & qMS6 & 6 & $22,576,747$ & $\mathrm{G} / \mathrm{T}$ & 0.07 & 9.12 & Prupe.6G219600 & $\mathrm{N}$-acetyl-I-glutamate synthase 1 \\
\hline \multirow[t]{6}{*}{ Chilling requirement } & qCR1 & 1 & $41,831,614$ & $C / T$ & 0.06 & 10.66 & Prupe.1G506600 & Galactosyltransferase family protein \\
\hline & qCR2 & 2 & $27,316,884$ & $A / G$ & 0.06 & 8.91 & Prupe.2G267100 & Homolog of yeast autophagy $18 \mathrm{C}$ \\
\hline & qCR3 & 3 & $26,702,108$ & $A / G$ & 0.06 & 9.47 & Prupe.3G303800 & Aldolase-type TIM barrel family protein \\
\hline & qCR5 & 5 & $6,157,490$ & $\mathrm{G} / \mathrm{C}$ & 0.06 & 10.08 & Prupe.5G056900 & Glutamate dehydrogenase 2 \\
\hline & qCR7 & 7 & $14,222,137$ & $\mathrm{~T} / \mathrm{G}$ & 0.05 & 8.37 & Prupe.7G117400 & O-acyltransferase (WSD1-like) family \\
\hline & qCR8 & 8 & $14,129,632$ & $\mathrm{~A} / \mathrm{C}$ & 0.07 & 9.20 & Prupe.8G112500 & Target of rapamycin \\
\hline \multirow[t]{5}{*}{ Leaf gland } & qLG1 & 1 & $44,394,635$ & T/G & 0.12 & 8.57 & Prupe.1G543300 & $\begin{array}{l}\text { WWE protein-protein interaction } \\
\text { protein }\end{array}$ \\
\hline & qLG2 & 2 & 718,751 & $\mathrm{G} / \mathrm{T}$ & 0.05 & 9.34 & Prupe.2G007800 & Tetratricopeptide repeat protein \\
\hline & qLG4 & 4 & $4,205,706$ & $C / T$ & 0.27 & 8.07 & Prupe.4G085700 & Unknown protein \\
\hline & qLG6 & 6 & $18,913,276$ & $A / G$ & 0.13 & 7.64 & Prupe.6G182200 & Ethylene-responsive transcription factor \\
\hline & qLG7 & 7 & $13,965,851$ & $C / T$ & 0.08 & 14.31 & Prupe.7G114100 & Cytochrome P450, family 94 \\
\hline \multirow[t]{5}{*}{ Leaf width } & qLW1 & 1 & $12,455,804$ & $A / C$ & 0.06 & 8.36 & Prupe.1G157000 & Major facilitator superfamily protein \\
\hline & qLW2 & 2 & $4,392,670$ & T/A & 0.06 & 17.26 & Prupe.2G040200 & Mitochondrial transcription terminator \\
\hline & qLW4 & 4 & $24,983,387$ & $\mathrm{~T} / \mathrm{C}$ & 0.11 & 11.00 & Prupe.4G286000 & Leucine-rich repeat-containing protein \\
\hline & qLW6 & 6 & $18,746,810$ & $\mathrm{G} / \mathrm{A}$ & 0.37 & 20.59 & Prupe.6G180800 & Thioesterase superfamily protein \\
\hline & qLW7 & 7 & $3,572,666$ & T/A & 0.06 & 15.73 & Prupe.7G022800 & $20 S$ proteasome alpha subunit G1 \\
\hline \multirow[t]{3}{*}{ Bottom leaf carotenoid } & qBLC1 & 1 & $47,191,519$ & $\mathrm{G} / \mathrm{A}$ & 0.05 & 26.20 & Prupe.1G580200 & Cytochrome P450, family 98 \\
\hline & qBLC2 & 2 & $19,689,635$ & $\mathrm{~T} / \mathrm{G}$ & 0.06 & 26.17 & Prupe.2G140100 & PLATZ transcription factor \\
\hline & qBLC6 & 6 & $21,730,489$ & $C / T$ & 0.05 & 14.14 & Prupe.6G208900 & Agamous-like MADS-box protein \\
\hline
\end{tabular}


Table 1 continued

\begin{tabular}{|c|c|c|c|c|c|c|c|c|}
\hline Traits & QTL & Chromosome & Position & Marker & MAF & $-\log P$ & Gene & Annotation \\
\hline \multirow[t]{4}{*}{ Bark Chl a/b } & $\mathrm{qBC2}$ & 2 & $18,030,212$ & $\mathrm{~A} / \mathrm{C}$ & 0.08 & 10.30 & Prupe.2G124000 & $\begin{array}{l}\text { Phosphoglycerate mutase family } \\
\text { protein }\end{array}$ \\
\hline & $\mathrm{qBC} 3$ & 3 & $18,444,624$ & $\mathrm{G} / \mathrm{A}$ & 0.08 & 11.83 & Prupe.3G165400 & NADH-ubiquinone dehydrogenase \\
\hline & $\mathrm{qBC} 4$ & 4 & $13,926,275$ & $\mathrm{~T} / \mathrm{C}$ & 0.05 & 11.97 & Prupe.4G221700 & Glycosyl hydrolase 9B8 \\
\hline & $\mathrm{qBC5}$ & 5 & $2,488,936$ & $\mathrm{~T} / \mathrm{A}$ & 0.09 & 13.67 & Prupe.5G022400 & $\begin{array}{l}\text { Steroidogenic acute regulatory } \\
\text { protein } 1\end{array}$ \\
\hline Bark carotenoid content & $\mathrm{qBCC} 3$ & 3 & $23,932,892$ & $\mathrm{~A} / \mathrm{G}$ & 0.1 & 7.36 & Prupe.3G248900 & RING/U-box superfamily protein \\
\hline \multirow[t]{5}{*}{ Top leaf anthocyanin } & qTLA2 & 2 & $21,308,922$ & $\mathrm{~T} / \mathrm{G}$ & 0.05 & 9.84 & Prupe.2G162400 & Phosphomannomutase \\
\hline & qTLA4 & 4 & $23,418,535$ & $\mathrm{G} / \mathrm{T}$ & 0.45 & 9.65 & Prupe.4G278400 & Unknown protein \\
\hline & qTLA5 & 5 & $17,879,238$ & $\mathrm{G} / \mathrm{A}$ & 0.05 & 7.82 & Prupe.5G236100 & Unknown protein \\
\hline & qTLA6 & 6 & $27,563,704$ & $\mathrm{~A} / \mathrm{T}$ & 0.39 & 11.44 & Prupe.6G305100 & Isopenicillin-N epimerase \\
\hline & qTLA7 & 7 & $17,385,707$ & $\mathrm{~T} / \mathrm{C}$ & 0.05 & 11.02 & Prupe.7G173400 & Winged-helix transcription factor \\
\hline \multirow[t]{5}{*}{ Middle leaf anthocyanin } & qMLA2 & 2 & $19,136,556$ & $\mathrm{G} / \mathrm{T}$ & 0.05 & 20.53 & Prupe.2G133600 & ELMO/CED-12 family protein \\
\hline & qMLA3 & 3 & $15,872,751$ & $\mathrm{~A} / \mathrm{G}$ & 0.07 & 23.77 & Prupe.3G145200 & PR5-like receptor kinase \\
\hline & qMLA4 & 4 & $15,695,604$ & $\mathrm{~A} / \mathrm{T}$ & 0.07 & 20.49 & Prupe.4G239100 & Cytochrome P450, family 71 \\
\hline & qMLA5 & 5 & $13,984,926$ & $\mathrm{~A} / \mathrm{G}$ & 0.06 & 13.00 & Prupe.5G157400 & Galactose oxidase repeat protein \\
\hline & qMLA6 & 6 & $23,930,128$ & $\mathrm{~A} / \mathrm{T}$ & 0.05 & 8.03 & Prupe.6G240500 & Unknown protein \\
\hline
\end{tabular}

always higher than that in any of the round-type fruit accessions $(n=25)$ (Fig. $2 \mathrm{~g})$. The ratio of supported reads for the two alleles of Prupe.6G290900 was similar in round-type accession (50\% v 50\%), while it was significantly different $(28 \% \mathrm{v} 72 \%)$ in flat-type accession at 80 DAFB based on RNA-seq (Fig. 2h), suggesting an allelespecific expression pattern of Prupe.6G290900 in flat-type accessions. To validate this discovery, we overexpressed Prupe.6G290900 in round-type wild-type tomato (Fig. 2i), and we observed that in three independent overexpressing lines of Prupe.6G290900 $(n=3)$, all fruits were flatter than wild-type fruits (Fig. $2 \mathrm{j}, \mathrm{m}$ ) and were flatter at each sampled time during the fruit development process (Fig. $2 \mathrm{k}$ ). The fruit shape index of these overexpressing lines was significantly smaller than that of the wild type (Fig. $2 \mathrm{~m}$ ), and the fruit shape index was negatively proportional to the relative expression of Prupe.6G290900 (Fig. 2l, m). Based on the locations of these two inversions, we designed two pairs of primers to genotype the 64 randomly selected accessions in our resource nursery. We found that all flat-type accessions $(n=32)$ showed an $\sim 300$ bp DNA product with the primer pair upstream of this inversion; however, none of the round-type accessions $(n=32)$ showed this $\sim 300 \mathrm{bp}$ DNA product (Fig. S5). Moreover, using a primer pair downstream of this inversion, we found that an $\sim 500 \mathrm{bp}$ PCR product could be amplified from all 32 flat-type accessions, and no DNA fragment could be amplified in any of the 32 round-type accessions (Fig. S6). This study showed that Prupe.6G290900, not Prupe.6G323700 (the expression level of this gene was not related to fruit shape, Fig. S7), was the best candidate gene for the flat-shape trait of peach. We hypothesized that a cis-element from the promoter of Prupe.6G323700 was transferred downstream of Prupe.6G290900 as an enhancer to activate its expression. We considered Prupe.6G323700 the best candidate gene involved in the premature abortion of fruits trait described in a previous study ${ }^{33}$, as it was linked with flat-type peach and was a recessive trait, considering the important role of this gene in energy sensors ${ }^{38}$.

\section{GWAS on the nonacidity trait in peach fruit}

The peach fruit taste is a key internal quality that is determined by complex factors. Low acidity is dominant to high acidity, and a major gene was mapped to the beginning of scaffold Pp05 in previous studies ${ }^{17,39}$. Although the SNP/indel and SV datasets used for GWAS were all analyzed (Fig. S8), we identified a small indel (chr5:628,841; $P=1.3 \mathrm{e}-8$; T/TG) located upstream of the candidate Prupe.5G005400 gene that showed the strongest association with this trait, which is a different locus from the candidate reported by a previous study ${ }^{17}$ and encodes a switch subunit three protein. After careful analysis of the candidate interval, we identified a gene involved in fruit sugar content variance, as a sugar QTL and sugar/acidity QTL were previously reported in the 


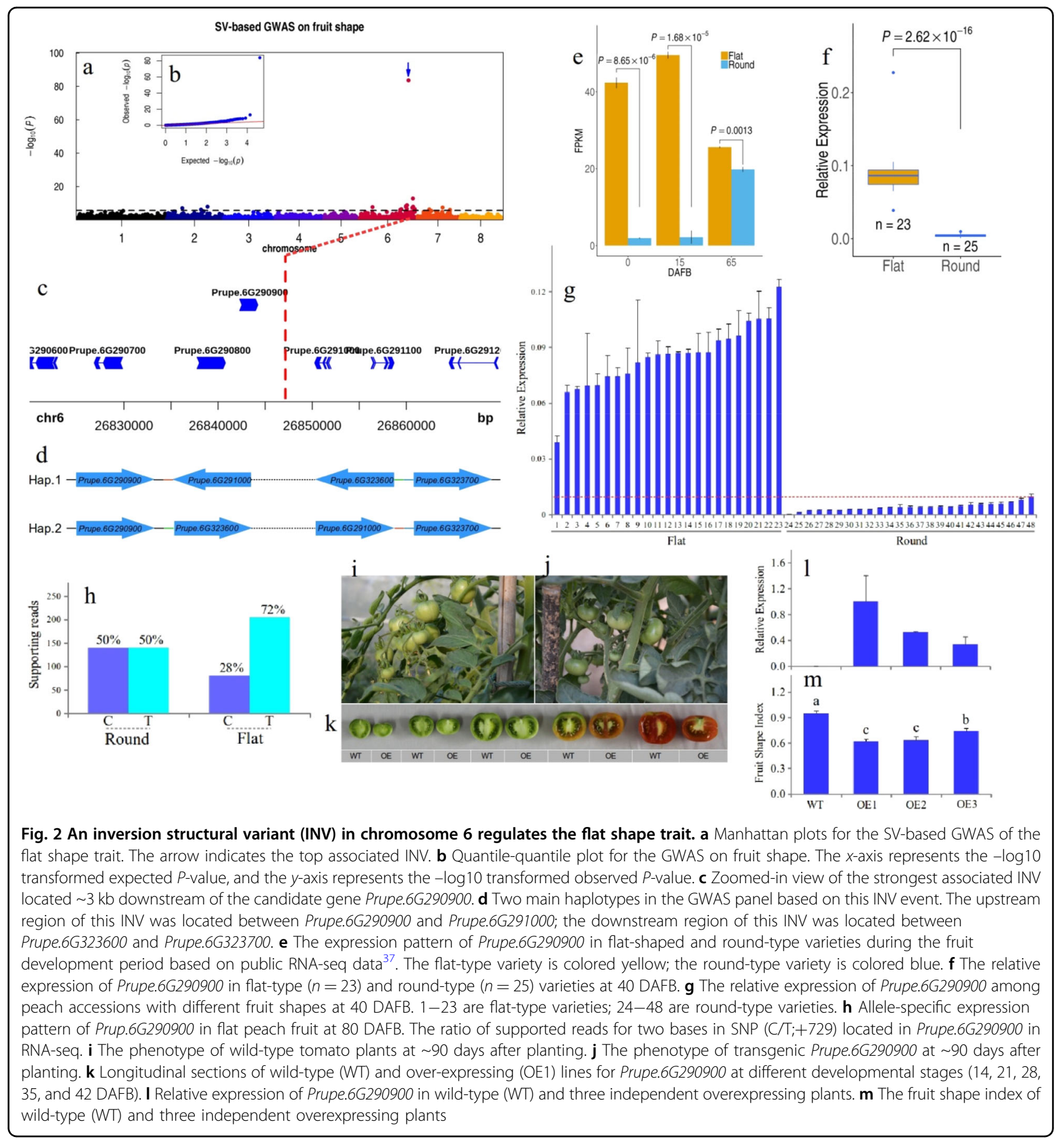

same interval ${ }^{40-42}$. We identified a single SNP (chr5:720,760; G/T; $P=5.00 \mathrm{e}-3$ ) in the third exon of Prupe.5G006300 (Fig. 3a), which encodes a sugar transport protein whose ortholog in Arabidopsis has been validated to control sugar transport ${ }^{43}$. This SNP leads to the conversion of acidic Q to $\mathrm{H}$ (Fig. 3c); this site was conserved from grass species to higher fruit-bearing plant species and from nematodes to humans (Fig. 3d). A protein crystal structure study of homologs in bacteria and mammals suggested that this site was one of the amino acids composing the binding site for sugar substrates $^{44,45}$. The conversion of this $Q$ site to A caused mostly functional loss of this gene and abrogated the sugar transport capacity of the cell in an in vitro experiment $^{44,45}$. Thus, this SNP may lead to functional loss of Prupe.5G006300 and generate a null allele. We analyzed the expression pattern of this gene-based on public data ${ }^{37}$ and found that it was relatively more highly expressed at 


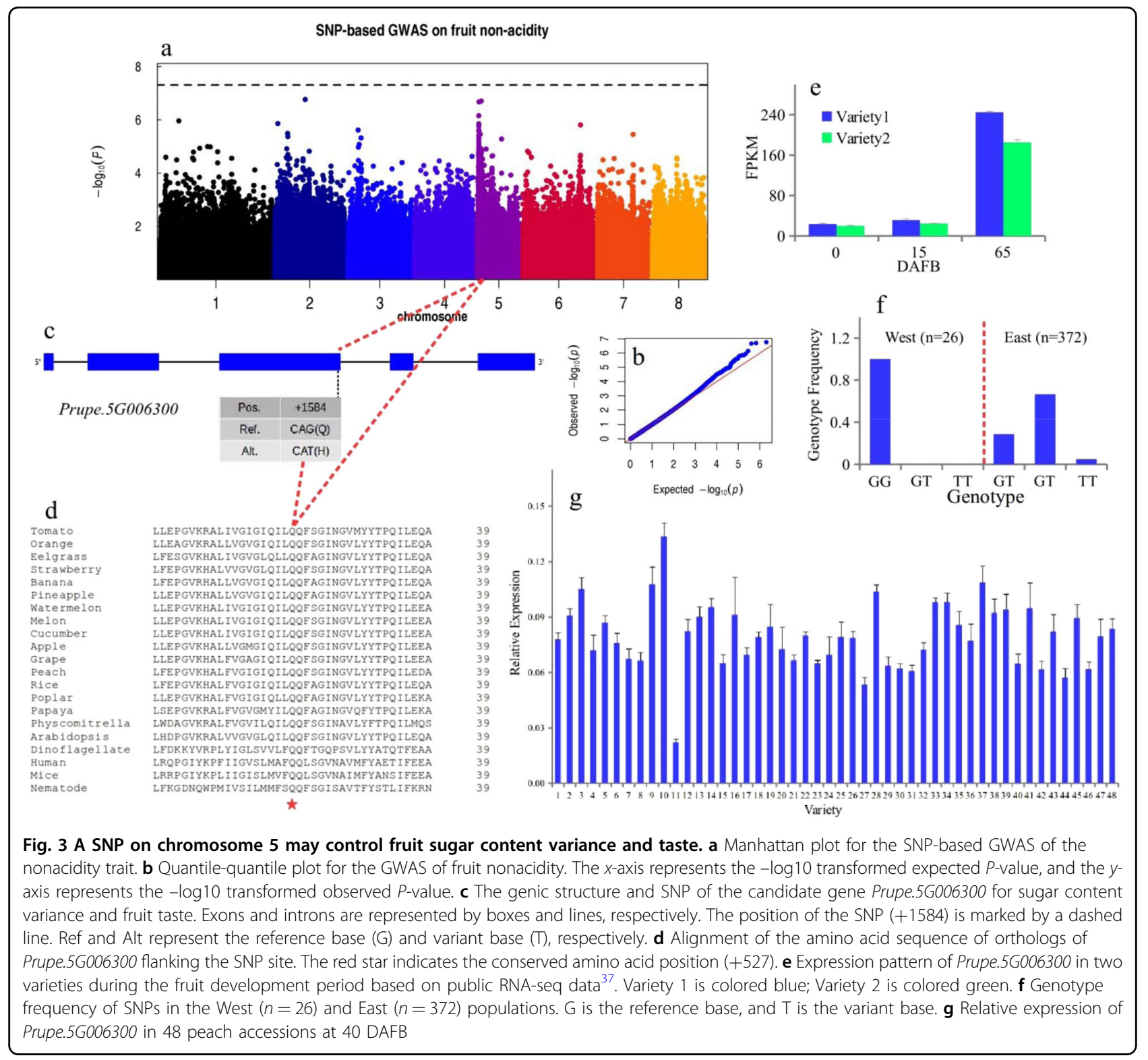

the fruit ripening stage in both varieties (65 DAFB) (Fig. $3 e)$, suggesting its role in enhancing the peach fruit sugar content at the mature stage. When we studied the genotype frequency in the West and East groups, we found that in the West population $(n=26)$, only individuals with the GG genotype were selected (Fig. 3f); these individuals had two copies of the functional allele for high sugar content. In contrast, in the East population $(n=372)$, individuals with the GT genotype were preferably selected (Fig. 3f). As all accessions in the West population in this study showed normal acidity $(\mathrm{pH}<4)$ and most accessions in the East population (79\% in this study) were nonacidic $(\mathrm{pH}>4)$, accessions with relatively high acidity and a high sugar content were selected in the West, and accessions (TT) with a low sugar content were selected elsewhere
(Fig. 3f; <5\%). The relative expression level of Prupe.5G006300 at 40 DAFB was similar across 48 peach accessions (Fig. 3g), suggesting that this gene is not differentially regulated at the transcriptional level.

\section{GWAS on the fruit development period}

The fruit development period and maturity date traits are key agronomic traits that determine the harvest and shipping time in peach production. Previous studies have shown that a major gene was mapped to the middle part of scaffold Pp04 in the peach genome based on linkage analysis $^{46}$, and one NAC candidate gene, Prupe.4G186800, with nine base insertions in its last exon was proposed $^{46}$. In the SNP-based GWAS approach, we identified SNPs with the strongest association signals 
(chr3:24,599,761; C/T; $P=8.00 \mathrm{e}-11$ ) where no suitable candidate genes were identified for this trait (Fig. S9a). We further performed an SV-based GWAS to try to discover candidate genes. An $\sim 400 \mathrm{bp}$ DEL variant with the strongest association signal $(\mathrm{chr} 4: 11,127,010)$ was located $\sim 10 \mathrm{~kb}$ upstream of Prupe.4G187100 (Fig. 4a, b), which encodes an NAC transcription factor whose ortholog in tomato was the NOR (non ripening) gene closely associated with fruit ripening ${ }^{47}$. A recent study confirmed the function of this gene in fruit ripening in peach ${ }^{48}$. This candidate gene was located on a different scaffold from the SNP with the strongest association discovered by standard SNP-based GWAS in this study (Fig. S7a); however, it was only $\sim 10 \mathrm{~kb}$ from the strongest signal based on the SV-based GWAS (Fig. 4a, b). A further study suggested that this $\sim 400 \mathrm{bp}$ DEL variant is within a complex genome rearrangement interval. Haplotyping analysis suggested that there were three main haplotypes (Fig. 4c) based on the large structural variant. Hap. 1 is the reference sequence $(\sim 2 \mathrm{~kb})$, and Hap. 2 is the rearrangement result of an $\sim 400 \mathrm{bp}$ DEL (right dashed line), another $\sim 190$ bp DEL (left dashed line), and an 130 bp INS (two left rectangles), in contrast to the reference genome. This $\sim 130$ bp INS included an $\sim 100$ bp sequence (the middle yellow rectangle) identical to the flanking downstream sequence at the insertion site (the right yellow rectangle). Thus, Hap. 2 is $\sim 1,550$ bp in length. Hap. 3 is the tandem repeat of Hap. 2 and has an $\sim 3 \mathrm{~kb}$ length (Fig. 4c). After analyzing 187 accessions with phenotypic data, we found that accessions with Hap. $1(n=47)$ had significantly longer fruit development periods than those with Hap. $2\left(n=58 ; P=1.62 \times 10^{-7}\right)$ and Hap. $3(n=82$; $\left.P<2 \times 10^{-16}\right)$ (Fig. 4e). Accessions with Hap. $2(n=58)$ also had significantly longer fruit development periods than those with Hap. $3\left(n=82 ; P<2 \times 10^{-16}\right)$ (Fig. 4e). We later detected the expression level of Prupe.4G187100 in 31 accessions at 40 DAFB at the population level (Fig. 4f). The expression level of this gene in the population with Hap. $1(n=5)$ was significantly lower than those in the population with Hap. $2(n=16 ; P=0.0109)$ and Hap. $3\left(n=10 ; P=4.56 \times 10^{-4}\right)$ (Fig. 4f). The expression level of this gene in the population with Hap. $2(n=16)$ was significantly lower than that in the population with Hap. 3 $\left(n=10 ; P=9.46 \times 10^{-8}\right)$ (Fig. 4f). At the individual level, the expression pattern of Prupe.4G187100 was significantly different across 31 peach accessions (Fig. 4i); most accessions with a short fruit development period had the highest expression level, and most accessions with a long fruit development period had the lowest expression level, suggesting that this gene is regulated at the transcriptional level. However, some accessions, such as 4, 14, 20, 21, 22, and 23, showed an opposite trend. This suggested that other loci were involved in this trait, as it is a QTL controlled by many gene $\operatorname{loci}^{49}$. When grouping accessions based on haplotypes, we found that in accessions with Hap. 1, the expression level of this gene was the lowest; in accessions with Hap. 2, the expression level of this gene was the median; and in accessions with Hap. 3, the expression level of this gene was the highest (Fig. 4j). The expression pattern of Prupe.4G187100 during fruit development was analyzed based on public data ${ }^{37}$, and we found that the expression level of Prupe.4G187100 was very high at the fruit ripening stage (Fig. 4g; 65 DAFB), suggesting that this gene is closely associated with fruit ripening. Furthermore, using transcriptome and genome data of the same accession $(n=2$; heterozygous Hap. 1/ Hap. 3), we found a clear allele-specific expression pattern of Prupe.4G187100 at the mature fruit stage (Fig. 4h; 99\% v 1\%; $80 \mathrm{DAFB}$ ), suggesting that there is a cis-element regulating its expression. This complex structural rearrangement interval may be the best candidate for the underlying cause of this allele-specific expression pattern. In a previous study, a homozygous $\sim 26.6 \mathrm{~kb}$ DEL located 700 bp upstream of Prupe.4G187100, which includes the complex structural rearrangement interval in the peach cultivar "Venus", abolished fruit ripening ${ }^{50}$, further indicating that this complex structural rearrangement was associated with peach fruit development and maturity. The ortholog in the apple was also confirmed to be closely associated with the fruit development period in a previous GWAS $^{51}$; this interval showed collinearity in apple, peach, apricot, and berry ${ }^{52}$. Thus, these genes may control fruit development using a conserved mechanism in these species. The GWAS results on the maturity date trait were the same as those on the development period trait (Fig. S10).

\section{GWAS on the double flower trait}

Double flowers with extra petals are important for artificial selection because of their attractive appearance and commercial value in several ornamental plants, such as peach. Two distinct loci were described as the underlying genetic causes for the double flower traits in peach. The first locus responsible for a recessive trait $(\mathrm{Dl} / \mathrm{dl})$ for double flowers was described by Lammerts ${ }^{53}$ and was mapped to chromosome $2^{17,54}$. The second locus, identified as a single dominant gene ( $\mathrm{Dl} 2 / \mathrm{dl} 2)$, which was first described by Beckman et al. ${ }^{55}$, was assigned to chromosome 6 and identified as a TOE-type AP2 gene. Deletion of the miR172 target site in this gene is responsible for the dominant double-flower trait in Rosaceae ${ }^{56,57}$. However, to date, the $\mathrm{Dl}$ gene is still controversial. In this study, we first performed SNP-based GWAS and identified the top associated SNP (chr6:21,609,914; A/G; 3.52e-40; Fig. S11), which was located on a different scaffold from the Dl gene, which was located on chromosome 2. We later performed SV-based GWAS and discovered that the top associated signal was in the promoter of Prupe.2G237700 


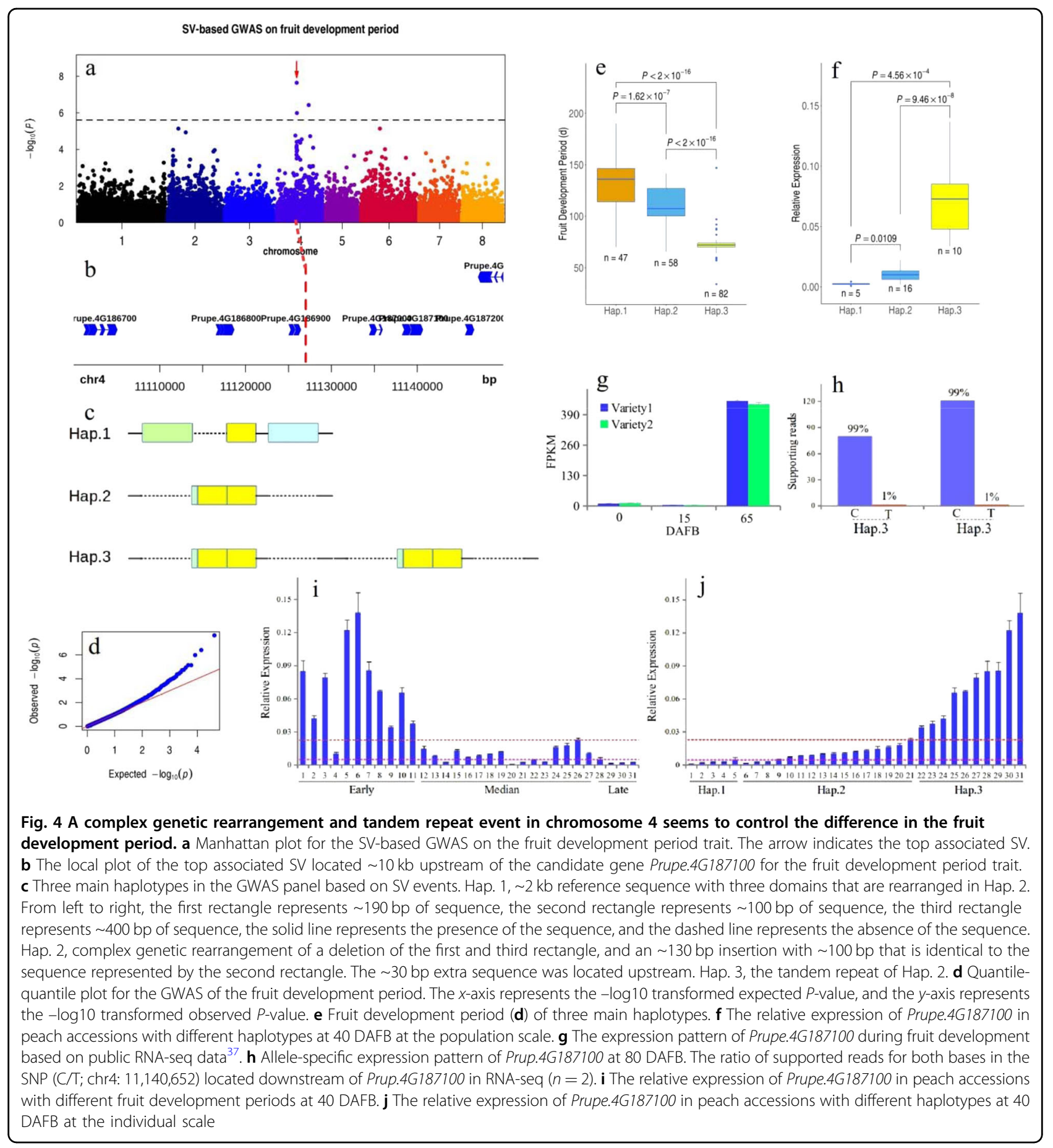

(chr2: 25,860,343; 9.99e-83; Fig. 5a, b), which is a different gene locus from the candidate reported by a previous study ${ }^{17}$ and annotated as a 70 amino acid peptide without a functional domain. Using 1860 bp of genomic sequence as a query for blasting the NCBI nucleotide collection (nr/nt) dataset (https://blast.ncbi.nlm.nih.gov/ Blast.cgi), we discovered that this gene is actually a noncoding RNA that transcribes the miR172d precursor (yellow-colored sequence in Fig. 5e). The strongest associated signal (chr2: 25,860,343) was only $333 \mathrm{bp}$ from mature miR172d (Fig. 5d; blue-colored sequence in Fig. 5e). After analyzing all peach accessions with a double flower phenotype $(n=7)$, we identified two independent insertion events within the promoter of Prupe.2G237700 and a total of three main haplotypes based on a large insertion SV (Fig. 5d). Hap. 3 included the top associated 

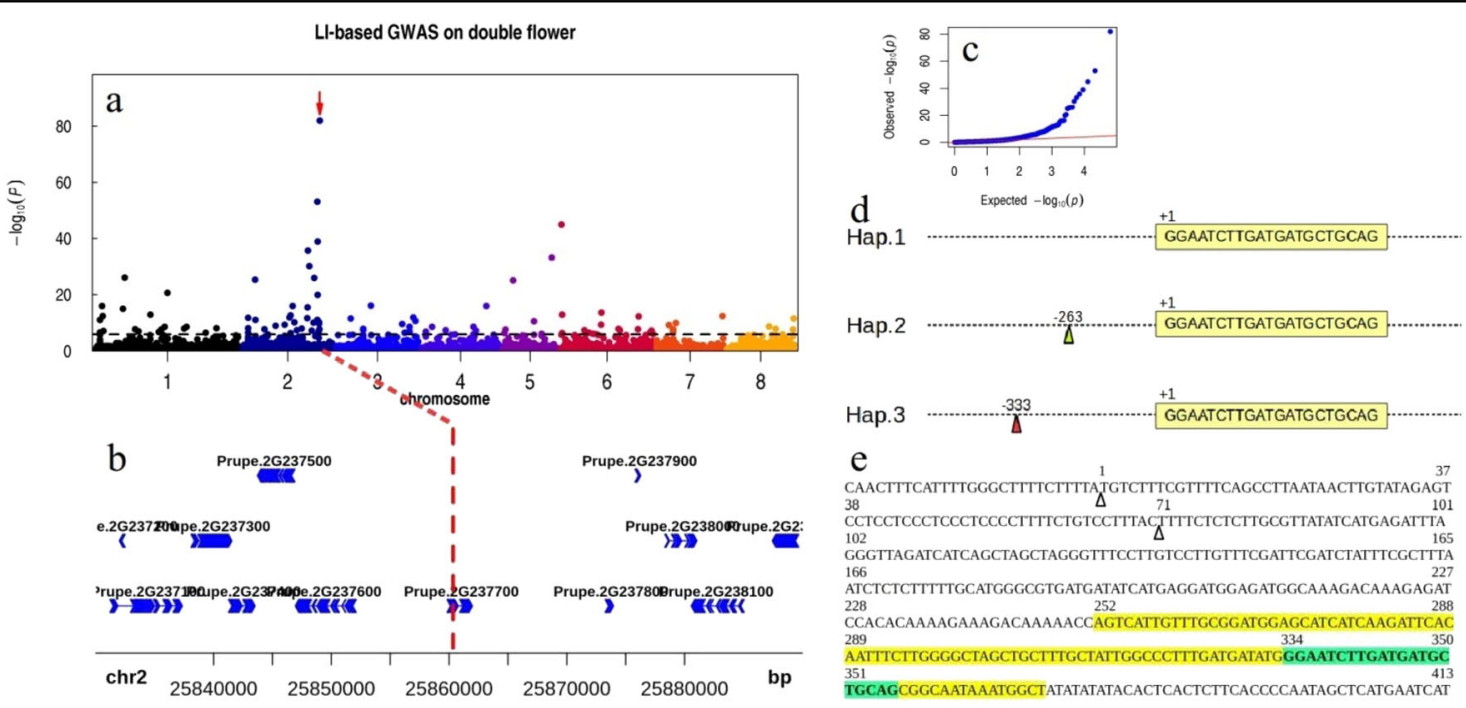

Fig. 5 Two independent transposable element insertion events in chromosome 2 seem to be the underlying genetic basis responsible for the double flower phenotype. a Manhattan plot for the LI-based GWAS of the double flower trait. The arrow indicates the top associated LI. $\mathbf{b}$ The local plot of the top associated LI located upstream of the promoter of the candidate gene Prupe.2G237700 for the double flower trait. c Quantilequantile plot for the GWAS on the double flower trait. The $x$-axis represents the -log 10 transformed expected $P$-value, and the $y$-axis represents the - $\log 10$ transformed observed $P$-value. $\mathbf{d}$ Three main haplotypes based on transposable element insertion. The sequence represents mature miR172d, and the dashed line represents its flanking sequence. Hap. 1, the reference sequence; Hap. 2, an 1.2 kb transposable element inserted at -263 bp (blue triangle) from mature miR172d; Hap. 3, an $\sim 5.5 \mathrm{~kb}$ transposable element inserted at $\sim-333 \mathrm{bp}$ (red triangle) from mature miR172d. e Sequence analysis of the candidate gene Prupe.2G237700. Two independent insertion sites of the transposable element are represented by triangles; the $\sim 120$ bp precursor of miR172d is colored yellow, and the mature miR172d is colored blue

signal (chr2: $25,860,343$ ), which is an $\sim 5.5 \mathrm{~kb}$ transposable element with an $\sim 250$ bp LRT (Fig. S12). Hap. 2 harbored an $\sim 1.2 \mathrm{~kb}$ transposable element without an LRT (chr2: 25,860,413; $263 \mathrm{bp}$ from the mature miR172d) (Fig. S13). These two independent insertion events may prevent the transcription of the miR172d precursor and result in decreased levels of mature miR172d, and this decrease may lead to an increase in petal number, as the orthologs in other species and the target gene AP2 family were closely associated with petal number ${ }^{58-60}$.

\section{GWAS on the nectarine trait}

Nectarine in peach is a key agricultural character affecting both appearance and ecological adaptation. The nectarine trait is recessive to normal peach, and the major gene controlling this trait is the MYB gene Prupe.5G196100 in the middle of scaffold Pp05. A large insertion variant was discovered in the third exon of this gene and was shown to cause loss of function and the hairlessness phenotype ${ }^{6}$. To determine if this event was the only event responsible for all hairlessness phenotypes, we first performed a SNP-based GWAS and found that the top associated SNP (chr5:16,633,286; G/A; $P=4.90 \mathrm{e}$ -35) was located $\sim 700 \mathrm{~kb}$ downstream of this gene locus (Fig. S14a), where no proper candidate genes could be identified. We next performed an LI-based GWAS approach and showed that the strongest associated signal (chr5:15,893,169; $P=3.93 \mathrm{e}-15$ ) (Fig. S14d) was located within the third exon of this MYB gene (as previously reported $^{6}$ ). We further analyzed this insertion event in all nectarine accessions and found that all nectarine accessions from Gansu Province did not have this large insertion variant at this site. To determine other variants responsible for hairlessness traits in accessions from Gansu Province, we first performed a SNP-based GWAS on this trait, but we defined other nectarine accessions with known LIs (large insertion structural variants) as hairy peaches. We identified one SNP with the strongest association signal (chr5:15,893,290; A/G; $P=2.15 \mathrm{e}-43$ ) located in the third exon of the same gene, Prupe.5G196100 (Fig. 6a, c). Haplotyping analysis suggested that at least 3 haplotypes were responsible for the nectarine trait (Fig. 6d). Hap. 1 included the different and newly identified LI in the second exon of this gene in a landrace from Jiyuan city of Henan Province; Hap. 2 included the known LI in this study; and Hap. 3 included two newly identified SNPs for nectarine traits in accessions from Gansu Province. The SNP causing the conversion of the $Q_{250}$ amino acid to $R_{250}$ (Hap. 3) was located in a conserved position (Fig. 6e). Another observation indicated that Hap. $3\left(\mathrm{G}_{719} \mathrm{G}_{749}\right)$ was the cause of the hairlessness trait: all accessions with only one copy of the insertion (Hap. 2) were normal peach, but an accession with one copy of the insertion (Hap. 2) and $\mathrm{SNP}_{749}$ 


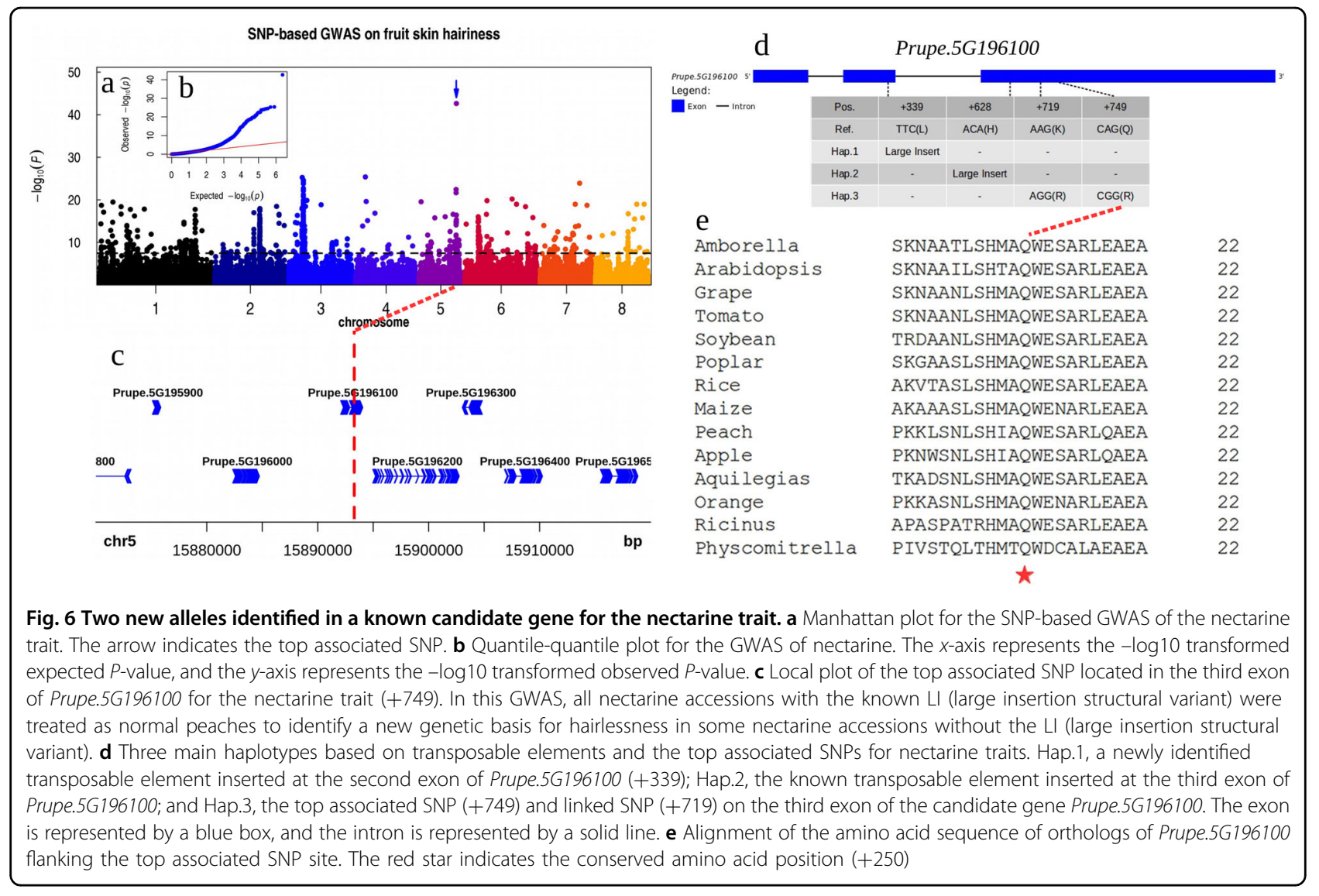

(Hap. 3) was a nectarine. Hap. 3 was only present in nectarines originating from Gansu Province. These observations suggested that the hairlessness trait originated from at least three independent mutation events.

\section{GWAS on the fruit flesh color trait}

Flesh color (white or yellow) in peach affects fruit nutritional value and consumer preference. White color is dominant to yellow color. The candidate gene Prupe.1G255500 was considered responsible for the trait, and three independent mutation events were considered responsible for the origin of yellow flesh ${ }^{5,61}$. In the present study, we first performed a SNP-based GWAS to try to relate the top associated SNPs to this gene. However, we discovered that the top SNP (chr1:29,627,336; T/G; $P=4.21 \mathrm{e}-10$ ) was $\sim 3 \mathrm{Mb}$ away from this gene (Fig. S15a). We later performed LI-based GWAS and identified the eighth strongest associated signal (chr1: 26,614,905; $P=1.21 \mathrm{e}-05)$ in the intron of Prupe.1G255500 (Fig. S15d), which was identified by a previous study ${ }^{5}$. A previous study suggested that the interval including the candidate gene was narrowed to $\sim 500 \mathrm{~kb}(25,842,123-26,865,123)$ on linkage group $1^{61}$; only the eighth strongest signal in this study was in this interval and thus discovered.

\section{Discussion}

A chromosome-grade genome assembly is valuable for identifying genetic variants and performing GWAS, providing new insights into the genetic architecture of key agronomic traits and genomic evolution ${ }^{62}$. In this study, we de novo assembled five species in the genus Prunus, demonstrated the utility of these genomes for the identification of structural variants, and provided a basis for functional genomics and comparative genomics in Prunus species.

GWAS is a versatile tool for identifying candidate genes and genetic architecture components for diseases and key agronomic and economic traits. Indeed, many studies have confirmed that this SNP-based approach was successful in discovering candidate genes ${ }^{9-13}$. However, this classic SNP-based approach was not very successful in peach, as few candidate genes and genetic architecture components were identified for traits in previous stu$\operatorname{dies}^{16,17}$ and in this study. To identify candidate genes, we had to adapt the traditional SNP-based GWAS approach for the fruit nonacidity and nectarine traits. After adjusting the GWAS approach, we identified a gene involved in fruit sugar content variance linked with nonacidity to some degree and a new allele for the nectarine trait from Gansu Province. Although selecting a 
sugar content variance-related gene in a GWAS of a nonacidity trait seemed improbable, this gene is located in a linkage interval harboring fruit sugar, acidity, and taste QTLs $^{40-42}$. Additionally, Prupe.5G006300 may be the candidate gene for this sugar QTL and may interact with genes associated with acidity to determine fruit taste. The allele frequency in the West and East populations suggested that this locus showed differential selection due to preferences for different tastes. For the nectarine trait from Gansu Province, a new allele of the candidate gene Prupe.5G196100 was identified. This finding suggested that the nectarine trait had multiple origins, similar to the fruit flesh color $^{5,61}$ and double flower traits in this study. This mechanism may be the universal mechanism for the adaptation of plants and natural or artificial selection.

We also used small indels as genetic markers to conduct GWASs, but this strategy was not successful in our study. The GWAS results were similar to those generated by SNPs. Additionally, no causal indel was identified for any trait. One possibility is that allelic heterogeneity is poor in this analysis, such as in the case of fruit flesh color ${ }^{5}$. The second possibility is that there was no casual indel for these traits or that the indel was not linked to any candidate genes or genetic architecture components. When traits were directly regulated by SVs identified by our SVbased GWAS (as described below), we found that it was difficult to relate the top associated SNPs with these SVs. One possible explanation is that the crossover and recombination rate could be extremely high in these flanking intervals if the SNP was not generated later than the SV or the flanking SNP was generated much later than the SV. In these two cases, some accessions with SVs will have no SNPs. The third possibility may be that very low $\mathrm{LD}$ in the peach genome prevents the linkage of the SNPs with the SVs; this case may have occurred in maize, which has a similar $L D$ value ${ }^{30}$. Furthermore, in some cases, the SNP is not linked with the candidate SV owing to a variety of mechanisms, such as mutation, genetic drift, and selection $^{20}$. In these cases, the candidate SV rather than the SNP was directly selected and enriched in a population, and the classic SNP-based GWAS will lose substantial detection power for key traits. SV-based GWASs will provide a new approach to identify candidate genes and genetic architecture components for key traits.

The genetic basis for agronomic traits in plant species varies; thus, only one approach to identifying candidate genes for key traits is limiting. A candidate SNP can be directly detected by traditional SNP-based GWASs, and as SVs are actually genetic marks similar to SNPs, candidate SVs may also be directly identified by SV-based GWASs. Thus, we used a combined SV-based GWAS approach to increase the success of identifying the underlying genetic architecture for traits. We provide evidence that SV-based GWASs can be used to identify candidate genes and genetic architecture components. Genotyping of DELs, DUPs, and INVs in a large population is feasible. Using the SV-based approach (DELs, DUPs, and INVs), we successfully identified a large inversion event responsible for flat shape in fruits and the premature abortion of fruits, as well as complex genomic rearrangements and tandem repeat events related to fruit development period and fruit maturity date traits. As the genotyping of INSs at a large population scale cannot be performed to date to our knowledge, we adapted a simplified genotyping approach, although it decreased the power of GWAS detection. Using this approach, we identified transposable element insertion events as the underlying causal factors for double flower, nectarine, and flesh color traits. All of these genes and candidate bases were lost in previous GWAS studies ${ }^{16,17}$, and we identified the top associated signals on these genes or very near them. These findings further indicated that INSs, especially transposable elements, are key genetic variants for many key agronomic traits, considering that transposable element events were responsible for three traits in this study and that transposable elements occur in the majority of the genome sequence.

We, fortunately, identified two independent transposable element events for double flowers, as only seven accessions with this phenotype were found in the 326 accessions in our GWAS panel. We concluded that the success was because no accessions were derived from crosses of edible peach and ornamental peach. Notably, the favorable traits of fruits and flowers were independently improved by breeders in the past. For the flat fruit shape trait, a previous study ${ }^{17}$ identified a SNP completely linked with this trait, which is $\sim 80 \mathrm{~kb}$ upstream of this INV. We inferred that this SNP was generated in conjunction with this INV as the same event and was thus linked with this trait as an INV, so these two variants were located in the same LD block. Our preliminary SNP-based GWAS did not identify this SNP as the top associated signal (Fig. S4a). This SNP was lost after filtering with $\mathrm{MAF}=0.05$ owing to the relatively small numbers of flattype peaches $(n=34)$. After we decreased this parameter to 0.04 , this SNP became the top associated signal (Fig. S16). Although a candidate $P p C A D$ gene was previously identified, including this $\mathrm{SNP}^{17}$, the expression pattern of this gene between flat-type and round-type fruit during the fruit development period cannot explain the fruit shape difference ${ }^{37}$. However, the physical position, expression pattern, and transgenic phenotype of Prupe.6G290900 suggested that it was the best candidate gene for fruit shape variance, although we cannot exclude the possibility that other genes affected by this INV are also associated with this trait. A Prupe.4G186800 gene was identified as the candidate gene for the fruit development period trait, and a small indel in the last exon was 
considered to be the causal element ${ }^{46}$. However, we found that this gene may not be a candidate gene for this trait based on three lines of evidence. First, the expression pattern suggested that this gene was not correlated with fruit ripening (data not provided). Second, the transgenic phenotype of the two alleles (with and without small indels) was not associated with fruit ripening (data not provided). Third, the phylogenetic tree suggested that this gene may be related to the stress response ${ }^{46}$. However, these three lines of evidence (except the transgenic phenotype, for which the identification is under way) all supported Prupe.4G187100 as the candidate gene for development period traits. The association of Prupe.4G187100 with fruit ripening was also validated by a previous study ${ }^{48}$. Although a few genes and genetic architecture components were identified for some traits, the genetic architecture of many traits in this study was still not identified by the SV-based GWAS approach. Other types of variants, such as epigenetic footprints, may be the underlying genetic basis for some traits. Allelic heterogeneity may also hinder the discovery of candidate genes and genetic architecture components. The environmental effect on phenotypes was not uniform. Further effort is still needed to identify and validate more candidate genes for these key traits. For INS-based GWAS, major efforts are also needed to develop tools and software for genotyping this SV at a large population scale.

In summary, we de novo assembled five species in the genus Prunus and generated a useful sequence dataset, which will help promote Prunus functional genomics and comparative genomics in fruit species in the future. The identified candidate genes and genetic architecture components by GWASs may provide targets for molecular marker selection and the improvement of key traits. Additionally, this comprehensive GWAS approach could be used in future deep sequencing studies to more precisely identify candidate genes and genetic architecture components for diseases and key traits in plants, animal species, and humans.

\section{Materials and methods}

\section{Genome sequencing}

Heterozygous diploid trees of five Prunus species were grown in our fruit field (Tai'an, Shandong, China). Fresh leaflets were collected and stored in liquid nitrogen until DNA extraction and sequencing. The long-read, short-read, and $\mathrm{Hi}-\mathrm{C}$ libraries were all prepared and sequenced at Annoroad Genomics (Beijing, China) (http://en.annoroad.com/) following the manufacturer's standard protocols. The $20 \mathrm{~kb}$ PacBio library was prepared and sequenced on a PacBio Sequel II using P6-C4 chemistry. A short-insert library with $400 \mathrm{bp}$ inserts and fragment sizes of $300-500 \mathrm{bp}$ was constructed for the $\mathrm{Hi}-\mathrm{C}$ library and sequenced with an Illumina HiSeq X-Ten platform.

\section{Genome assembly and quality assessment}

Filtered PacBio subreads were first assembled with Falcon $(\mathrm{v} 0.4)^{63}$ with a genome size estimation of $250 \mathrm{Mb}$ as an input. One copy of the contigs from heterozygous regions was retained by using redundant sequences (v0.14a) with the following parameters: Prunus salicina: --identity 0.8 --overlap 0.8; Prunus persica: --identity 0.9 --overlap 0.9; Prunus armeniaca: --identity 0.8 --overlap 0.8; Prunus mira: --identity 0.9 --overlap 0.9; and Prunus davidiana: --identity 0.85 --overlap 0.8 . The resulting assembly was polished by aligning PacBio reads with Quiver ${ }^{64}$ followed by running Pilon (v1.20) ${ }^{65}$ with the Illumina short-read sequences. The reads from the $\mathrm{Hi}-\mathrm{C}$ library were aligned to the primary assembly using Bowtie $2^{66}$. The resulting bam files together with the contigs were used as input for Lachesis ${ }^{67}$ with the cluster number set to 9 and the remaining parameters set as default. The pseudoscaffolds constructed by Lachesis ${ }^{67}$ were split into bins of $100 \mathrm{~kb}$ and used to construct an interaction heatmap for validation and manual correction. The serial numbers of the chromosomes were manually adjusted in descending order of chromosome length (Chr1-longest; Chr8-shortest). Assembly completeness was assessed with BUSCO (Benchmarking Universal Single-Copy Orthologs) (v3.0.1) 28 using 1,375 plant ortholog groups (embryophyta v10) with default parameters.

\section{Genome annotation}

Genome annotation was based on full-length RNA-iso sequencing and homology-based and de novo prediction strategies. We generated RNA-Seq libraries from a mixture of leaves, phloem, fruit, and seeds and conducted full-length sequencing on the PacBio-Iso platform. The full-length transcripts were directly used to predict gene models with PASA ${ }^{68}$. Homology-based evidence was derived from protein sequences blasted against UniProt assemblies. De novo prediction of gene models was performed using Augustus ${ }^{69}$. Complete and nonredundant gene models were combined using EvidenceModeler ${ }^{70}$. Repeated annotation of the assemblies was based on homology and de novo prediction strategies. The homology-based prediction was performed with RepeatMasker $^{71}$ using the RepBase database ${ }^{72}$. De novo assembly of repeat sequences was conducted with RepeatModeler (http://www.repeatmasker.org/RepeatModler/), followed by the repeatMasker tool.

\section{Phylogenetic tree reconstructions and divergence time estimation}

Phylogenetic tree construction was performed based on 248 single-copy genes extracted from the gene family cluster analysis. We utilized MUSCLE software ${ }^{73}$ to perform protein alignments for each single-copy gene family with the default sets. The maximum likelihood tree was constructed using PhyML software ${ }^{74}$ with the default parameters. 
The divergence time of each node in the phylogenetic tree was estimated based on the BRMC model in the MCMCTree program from the PAML package ${ }^{75}$. The species divergence time calibration was based on TimeTree (http://timetree.org/).

\section{Sampling of $\mathbf{4 1 7}$ peach accessions}

A total of 417 accessions $(159+258)$ were characterized by whole-genome resequencing. Among them, 159 Prunus accessions were collected as described previously ${ }^{3,17,29}$, representing most ecotypes worldwide. The 258 peach accessions newly sequenced in this study were collected from various regions in China and planted in the Peach Germplasm Repository, Shandong Agricultural University, China. These 417 accessions included 5 accessions of $P$. davidiana, 11 accessions of $P$. ferganensis, 3 accessions of $P$. kansuensis, 8 accessions of $P$. mira, 1 accession of $P$. dulcis, 1 accession of $P$. tangutica, and 388 accessions of $P$. persica peach varieties. For the 258 newly sequenced accessions, the fresh leaf of a single individual was used for DNA extraction using the CTAB method, the insert size of the libraries was $350 \mathrm{bp}$, and the pairedend read length was $150 \mathrm{bp}$. The 258 samples were sequenced on the Illumina HiSeq 2500 platform.

\section{Phenotypic evaluation}

Nineteen agronomic traits were phenotyped in this study, including 11 qualitative and eight quantitative traits. The 11 qualitative traits included fruit shape (flat/round), flesh color (yellow/white), fruit hairiness (peach/nectarine), fruit nonacidity (high/low acidity), flesh adhesion (clingstone/freestone), flesh texture (hard/soft), flesh color around the stone (red/white), flower double (double/single), flower morphology (showy/nonshowy), male sterility (fertility/sterility), and leaf gland (globular/reniform). The eight quantitative traits included fruit development period, chilling requirement, leaf width, top leaf anthocyanin content, middle leaf anthocyanin content, bottom leaf carotenoid content, bark $\mathrm{Chl} \mathrm{a} / \mathrm{b}$, and bark carotenoid content. The fruit traits were evaluated using fully matured fruits. All traits were analyzed in at least five fruits, flowers, leaves, and bark sections, which were collected from the tree that was sequenced for each accession. All agronomic traits considered here were characterized based on previously published plant genetic resource evaluation criteria ${ }^{76}$. The traits for the 159 previously analyzed accessions were collected from previous papers ${ }^{16,17,29}$ and books ${ }^{77}$.

\section{SNP and indel calling}

In this study, we analyzed a total of 417 wholegenome sequences of 417 peach accessions. A total of 258 peach accessions were sequenced in this study. An additional 159 cultivated and wild peach accessions were downloaded from a public dataset (https://www. ncbi.nlm.nih.gov/sra). Then, 182 of 258 peach accessions were sequenced at a coverage of $\sim 20 \mathrm{x}$ (sequenced twice for each sample; each is $\sim 10 \mathrm{x}$ ), and 76 of 258 peach accessions were sequenced at a coverage of $\sim 10 x$. The 159 cultivated and wild peach accessions were sequenced unevenly from $3 x$ to $100 x$. The 159 public accessions were downloaded and mapped to the peach reference genome v2. $0^{3}$ with bwa-mem ${ }^{78}$, and the 258 accessions were mapped to the peach reference genome v2. $0^{3}$ with minimap ${ }^{79}$. The mapped results were sorted and filtered to remove PCR duplicates with samtools ${ }^{80}$ and Picard tools (https://broadinstitute.github.io/ picard/), respectively. The Genome Analysis Toolkit ${ }^{81}$ (GATK, version v3.8) was used to realign mapped sequences within the interval with INDEL. The Genome Analysis Toolkit ${ }^{81}$ (GATK, version v3.8) was used for jointly calling SNPs and small indels throughout the entire collection of 417 peach accessions with default parameters and recommended hard filtering. The SNP set was further filtered (--MAF 0.01, and --max-missing 0.75 ), and only the biallelic SNPs were retained for downstream analysis.

\section{SV calling and genotyping}

The 18 wild relative accessions and 73 accessions with a low depth of sequencing were excluded from downstream analyses, and 326 accessions were kept for SV calling and genotyping at a population scale. The 326 accessions were analyzed following the guidance of Smoove (https:// github.com/brentp/smoove) using input bam files generated as above. This pipeline was first used to call SVs for each accession to obtain a union of sites across all samples. These variant sites were used to genotype each accession, and the resulting single samples were merged to generate a raw variant dataset. This pipeline was used to call and genotype DELs, DUPs, and INVs in the 326 accessions. The Pindel tool (https://github.com/genome/ pindel) was used to call large insertions (LI, $>50 \mathrm{bp}$ ) in the 326 accessions, and the resulting variants were subsequently converted to vcf files. The pipeline for calling large insertions ( $\mathrm{LI},>50 \mathrm{bp}$ ) was first used for each accession and later merged with bcftools (https:// samtools.github.io/bcftools/bcftools.html).

\section{Characterization of this population}

The phylogenetic tree, PCA, population structure, and LD analyses were performed on 99,526 high-quality SNPs pruned with plink ${ }^{82}$ (--indep 505 2). The genetic distance between two given accessions was calculated with dnadist in Phylip ${ }^{83}$ (v3.96), and a neighbor-joining tree was constructed and visualized in itol (https://itol.embl.de/). The population genetic structure was examined via an expectation-maximization algorithm, as implemented in the program Admixture ${ }^{84}$. The number of assumed 
genetic clusters $\mathrm{K}$ ranged from 2 to 9, with 10,000 iterations for each run. PCA was performed using EIGENSTRAT tools ${ }^{85}$ and plotted with R software. LD decay was measured using PopLDdecay software ${ }^{86}$, which directly uses the Variant Call Format (VCF) file with many variants as input to produce the LD decay statistics and plot the LD decay graphs in a pipeline manner.

\section{Genome-wide association analysis}

A total of 999,567 high-quality SNPs in 417 samples, along with 152,210 indels, 21,416 DELs, DUPs, and INVs (further filtered with --MAF 0.05 and --max-missing 0.75), and 32,338 LIs in 326 samples (further filtered with --MAF 0.01 and --max-missing 0.75) were used to perform genome-wide association analysis on key traits. All GWASs were conducted with efficient mixed-model association expedited $(\text { EMMAX })^{87}$. Population structure was corrected using a kinship (K) matrix (Balding-Nichols matrix) estimated by the emmax-kin-intel package of EMMAX based on 999,567 SNPs. The first ten principal components of the PCA were included as the variance-covariance matrix for adjusting for population stratification based on 99,456 pruned SNPs. The genome-wide significance thresholds of all the traits were determined using the Bonferroni test. According to a nominal level of 0.05 , the threshold was determined by a threshold $P(P=0.05 / N, N$ is the number of markers). The GWAS based on SNPs and indels was performed on 417 samples, and these samples were used to calculate the $K$ matrix and variance-covariance matrix. The GWAS based on SVs was conducted on 326 samples, which were used to estimate the $\mathrm{K}$ matrix and variance-covariance matrix. To identify the variant associated with hairlessness in nectarines without the previously reported LI, we considered all nectarine accessions with the LI as normal peaches and the remaining nectarines without $\mathrm{LI}$ events as the other class of phenotypes. The Manhattan plots and local plots were generated with the Sushi ${ }^{88}$ and qqman ${ }^{89}$ packages.

\section{RNA-seq analyses}

To detect the expression level of candidate genes among different peach accessions, we sampled the fruit flesh of 48 peach cultivars at $\sim 40$ days after flowering. To analyze the allele-specific expression pattern of candidate genes, additional ripening fruit flesh tissue of eight peach cultivars at 80 days after full blooming was also collected. These fruit flesh tissues were used to extract RNA. A library with an insert size of $\sim 350$ bp was constructed, and $150 \mathrm{bp}$ paired-end reads were sequenced using HiSeq 4000. Reads were mapped onto the peach reference genome (V2.0) using hisat $2{ }^{90}$ with the default parameter. The resulting bam files were sorted, and PCR duplicates were removed with a Picard tool (https://broadinstitute.github. io/picard/). To analyze the allele-specific expression pattern of Prupe.6G290900 and Prupe.4G187100, we used accessions with heterozygous SNPs located at this gene locus to perform RNA-seq. We generated a total of $\sim 288 \mathrm{~Gb}$ of raw sequencing data for 48 peach accessions and $\sim 80 \mathrm{~Gb}$ for an additional 8 peach accessions. The allele-specific expression pattern of Prupe.4G187100 was visualized by $\mathrm{IGV}^{91}$. The transcripts of each sample and expression levels of the genes were built and estimated by using Stringtie ${ }^{92}$ with the default parameters. To estimate the FPKM value, we downloaded the data from NCBI (SRX3157062; SRX3157064-SRX3157074; SRX3157091 -SRX3157094; SRX3157147-SRX3157148). The reads were mapped against the peach reference (V2.0) with hisat $2^{90}$, and the FPKM value was estimated for the gene with StringTie ${ }^{92}$.

\section{qRT-PCR experiment}

The expression patterns of Prupe.6G290900, Prupe.6G323700, and Prupe.4G187100 in varieties at 40 DAFB were detected using qRT-PCR. Total RNA was extracted from whole fruit tissue with an EASYspin Plus Plant RNA Kit (Aidlab Biotech) and reverse transcribed with a PrimeScript RT Reagent Kit with gDNA Eraser (TaKaRa). Real-time PCR was performed in triplicate twice with SYBR Premix DimerEraser (Perfect Real Time) (TaKaRa). Prupe.6G163400 was used as an internal control to normalize gene expression. The results were analyzed according to the $\Delta \mathrm{Ct} \operatorname{method}^{93}$.

\section{Transgene experiments}

The CDS of Prupe.6G290900 was cloned into the PbI121 vector under the control of the $35 \mathrm{~S}$ promoter and transformed into tomato plants using the Agrobacteriummediated transformation approach with kanamycin as a selectable marker. Transgenic plants were validated by both PCR with genomic DNA and quantitative real-time RT-PCR. The transgenic lines (T0) of Prupe.6G290900 were photographed at various stages of fruit development.

\section{Statistical analyses}

One-way ANOVA $(P=0.05)$ was performed using $\mathrm{R}$ software and SPSS 22.

\section{Accession numbers}

The genome assemblies, annotation files, and raw data of DNA and RNA sequences have been deposited in the NCBI Sequence Read Archive under accession number PRJNA655343.

\section{Acknowledgements}

We are grateful to Lirong Wang (Zhengzhou Fruit Research Institute, Chinese Academy of Agricultural Sciences), who provided some valuable peach materials. We are also grateful to Sanwen Huang (Agricultural Genomics Institute at Shenzhen, Chinese Academy of Agricultural Sciences) and Zhonghua Zhang (Qingdao Agricultural University), who offered many valuable comments and suggestions for modifying this manuscript. We are also grateful to the anonymous reviewers for offering valuable comments and 
suggestions and for improving this manuscript. We thank all authors who participated in the studies mentioned in this manuscript. This work was supported by (1) National Key Research and Development Plan, grant No. 2018YFD1000104; (2) National Natural Science Foundation of China, grant No. 31872041; (3) Provincial Natural Science Foundation of Shandong, grant No. ZR2018MC023; (4) Shandong Province Agricultural Good Seed Project grant, No. 2020LZGC007 and 2020LZGC00702; and (5) Funding for major agricultural application technology innovation projects in Shandong Province.

\section{Author details}

${ }^{1}$ College of Life Sciences, Shandong Agricultural University, Tai'an 271018, People's Republic of China. ${ }^{2}$ State Key Laboratory of Crop Biology, Shandong Agricultural University, Tai'an 271018, People's Republic of China. ${ }^{3}$ College of Horticulture Science and Engineering, Shandong Agricultural University, Tai'an 271018, People's Republic of China. ${ }^{4}$ Shandong Collaborative Innovation Center for Fruit \& Vegetable Production with High Quality and Efficiency, Tai'an 271018, People's Republic of China. ${ }^{5}$ Yantai Institute of Coastal Zone Research, Chinese Academy of Sciences, Yantai 264003, People's Republic of China. ${ }^{6}$ College of Forestry, Shandong Agricultural University, Tai'an 271018, People's Republic of China

\section{Author contributions}

Q.P.T., L.L., and W.X. conceived and designed the experiments. Q.P.T., L.L., and W.X. performed the experiments and analyzed the data. The others provided technical support and theoretical support for this work. Q.P.T. wrote the paper, and Q.P.T., L.L., and W.X. revised the intellectual content of this paper. L.L. and W.X. supervised the project as co-corresponding authors.

\section{Conflict of interest}

The authors declare no competing interests.

Supplementary information The online version contains supplementary material available at https://doi.org/10.1038/s41438-021-00648-2.

Received: 1 March 2021 Revised: 18 May 2021 Accepted: 13 June 2021 Published online: 01 October 2021

\section{References}

1. Shulaev, V. et al. Multiple models for Rosaceae genomics. Plant Physiol. 147, 985-1003 (2008).

2. Faust, M. \& Timon, B. Origin and dissemination of peach. Hortic. Rev. (Am. Soc. Hortic. Sci.) 17, 331-379 (1995).

3. International Peach Genome, I. et al. The high-quality draft genome of peach (Prunus persica) identifies unique patterns of genetic diversity, domestication, and genome evolution. Nat. Genet. 45, 487-494 (2013).

4. Morgutti, S. et al. Changes in endopolygalacturonase levels and characterization of a putative endo-PG gene during fruit softening in peach genotypes with nonmelting and melting flesh fruit phenotypes. N. Phytologist 171, 315-328 (2006)

5. Falchi, R. et al. Three distinct mutational mechanisms acting on a single gene underpin the origin of yellow flesh in peach. Plant J. 76, 175-187 (2013).

6. Vendramin, E. et al. A unique mutation in a MYB gene cosegregates with the nectarine phenotype in peach. PLoS One 9, e90574 (2014).

7. Bielenberg, D. G. et al. Sequencing and annotation of the evergrowing locus in peach [Prunus persica (L.) Batsch] reveals a cluster of six MADS-box transcription factors as candidate genes for regulation of terminal bud formation. Tree Genet. Genomes 4, 495-507 (2008).

8. Chris Dardick, et al. PpeTAC1 promotes the horizontal growth of branches in peach trees and is a member of a functionally conserved gene family found in diverse plants species. Plant J. 75, 618-630 (2013)

9. Atwell, S. et al. Genome-wide association study of 107 phenotypes in Arabidopsis thaliana inbred lines. Nature 465, 627-631 (2010).

10. Yano, K. et al. Genome-wide association study using whole-genome sequencing rapidly identifies new genes influencing agronomic traits in rice. Nat. Genet. 48, 927-934 (2016).

11. Wang, X. et al. Genetic variation in ZmVPP1 contributes to drought tolerance in maize seedlings. Nat. Genet. 48, 1233-1241 (2016).
12. Tieman, D. et al. A chemical genetic roadmap to improved tomato flavor. Science 355, 391-394 (2017).

13. Ma, Z. et al. Resequencing a core collection of upland cotton identifies genomic variation and loci influencing fiber quality and yield. Nat. Gen. 50, 803-813 (2018)

14. Newell, M. A., Cook, D., Tinker, N. A. \& Jannink, J.-L. Population structure and linkage disequilibrium in oat (Avena sativa L.): implications for genome-wide association studies. Theor. Appl. Genet. 122, 623-632 (2011).

15. Bush, W. S. \& Moore, J. H. Chapter 11: Genome-wide association studies. PLoS Comput Biol. 8, e1002822 (2012).

16. Micheletti, D. et al. Whole-genome analysis of diversity and SNP-major gene association in peach germplasm. PLOS ONE 10, e0136803 (2015).

17. Cao, K. et al. Genome-wide association study of 12 agronomic traits in peach. Nat. Commun. 7, 13246 (2016).

18. Hangauer, MatthewJ., Vaughn, lanW. \& McManus, MichaelT. Pervasive transcription of the human genome produces thousands of previously unidentified long intergenic noncoding RNAs. PLoS Genet. 9, e1003569 (2013).

19. Mao, H. et al. A transposable element in a NAC gene is associated with drought tolerance in maize seedlings. Nat. Commun. 6, 8326 (2015).

20. Vonholdt, B. M. et al. Structural variants in genes associated with human Williams-Beuren syndrome underlie stereotypical hypersociability in domestic dogs. Sci. Adv. 3, e1700398 (2017).

21. Payer, L. M. et al. Structural variants caused by Alu insertions are associated with risks for many human diseases. Proc. Natl Acad. Sci. USA 114, E3984-E3992 (2017)

22. Flint-Garcia, S. A., Thornsberry, J. M. \& Buckler, E. S. Structure of linkage disequilibrium in plants. Annu Rev. Plant Biol. 54, 357-374 (2003).

23. Carbonell-Bejerano, P. et al. Catastrophic unbalanced genome rearrangements cause somatic loss of berry color in grapevine. Plant Physiol. 175, 00715 (2017).

24. Chen, K. et al. BreakDancer: an algorithm for high-resolution mapping of genomic structural variation. Nat. Methods 6, 677-681 (2009).

25. Ye, K., Schulz, M. H., Long, Q., Apweiler, R. \& Ning, Z. Pindel: a pattern growth approach to detect break points of large deletions and medium sized insertions from paired-end short reads. Bioinformatics 25, 2865-2871 (2009).

26. Chen, $X$. et al. Manta: rapid detection of structural variants and indels for germline and cancer sequencing applications. Bioinformatics 32, 1220-1222 (2015).

27. Jiang, F. et al. The apricot (Prunus armeniaca L.) genome elucidates Rosaceae evolution and beta-carotenoid synthesis. Hortic. Res. 6, 128 (2019).

28. Simão, F. A., Waterhouse, R. M., loannidis, P., Kriventseva, E. V. \& Zdobnov, E. M. BUSCO: assessing genome assembly and annotation completeness with single-copy orthologs. Bioinformatics 31, 3210-3212 (2015).

29. Cao, K. et al. Comparative population genomics reveals the domestication history of the peach, Prunus persica, and human influences on perennial fruit crops. Genome Biol. 15, 415 (2014).

30. Hufford, M. B. et al. Comparative population genomics of maize domestication and improvement. Nat. Genet. 44, 808-811 (2012).

31. Du, X. et al. Resequencing of 243 diploid cotton accessions based on an updated A genome identifies the genetic basis of key agronomic traits. Nat. Genet. 50, 796-802 (2018).

32. Lesley, J. W. A genetic study of saucer fruit shape and other characters in the peach. Proc. Am. Soc. Hortic. Sci. 37, 218-222 (1940).

33. Dirlewanger, E. et al. Development of a second-generation genetic linkage map for peach [Prunus persica (L.) Batsch] and characterization of morphological traits affecting flower and fruit. Tree Genet. Genomes 3, 1-13 (2006).

34. Picañol, R. et al. Combining linkage and association mapping to search for markers linked to the flat fruit character in peach. Euphytica 190, 279-288 (2013).

35. Liu, J., Van Eck, J., Cong, B. \& Tanksley, S. D. A new class of regulatory genes underlying the cause of pear-shaped tomato fruit. Proc. Natl Acad. Sci. USA 99 13302-13306 (2002)

36. Ramon, M. et al. The hybrid four-CBS-Domain KIN $\beta y$ subunit functions as the canonical $\gamma$ subunit of the plant energy sensor Sn RK 1. Plant J. 75, 11-25 (2013).

37. Guo, J. et al. Comparative transcriptome and microscopy analyses provide insights into flat shape formation in peach (Prunus persica). Front. Plant Sci. 8, 2215 (2018).

38. Broeckx, T., Hulsmans, S. \& Rolland, F. The plant energy sensor: evolutionary conservation and divergence of SnRK1 structure, regulation, and function. J. Exp. Bot. 67, 6215-6252 (2016). 
39. Boudehri, $\mathrm{K}$. et al. Phenotypic and fine genetic characterization of the D locus controlling fruit acidity in peach. BMC Plant Biol. 9, 59 (2009).

40. Dirlewanger, E. et al. Mapping QTLs controlling fruit quality in peach (Prunus persica (L.) Batsch). Theor. Appl. Genet. 98, 18-31 (1999).

41. Dirlewanger, E. et al. New detection of QTLs controlling major fruit quality components in peach. VI Int. Peach Symp . 713, 65-72 (2005). January.

42. Zeballos, J. L. et al. Mapping QTLs associated with fruit quality traits in peach [Prunus persica (L.) Batsch] using SNP maps. Tree Genet. Genomes 12, 37 (2016).

43. Wormit, A. et al. Molecular identification and physiological characterization of a novel monosaccharide transporter from Arabidopsis involved in vacuolar sugar transport. Plant Cell 18, 3476-3490 (2006).

44. Sun, L. et al. Crystal structure of a bacterial homologue of glucose transporters GLUT1-4. Nature 490, 361-366 (2012)

45. Nomura, N. et al. Structure and mechanism of the mammalian fructose transporter GLUT5. Nature 526, 397-401 (2015).

46. Pirona, R. et al. Fine mapping and identification of a candidate gene for a major locus controlling maturity date in peach. BMC Plant Biol. 13, 166 (2013).

47. Giovannoni, J. J. et al. Molecular genetic analysis of the ripening-inhibitor and non-ripening loci of tomato: a first step in genetic map-based cloning of fruit ripening genes. Mol. Gen. Genet. MGG 248, 195-206 (1995).

48. Lü, P. et al. Genome encode analyses reveal the basis of convergent evolution of fleshy fruit ripening. Nat. Plants 4, 784-791 (2018).

49. Eduardo, I. et al. QTL analysis of fruit quality traits in two peach intraspecific populations and importance of maturity date pleiotropic effect. Tree Genet. Genomes 7, 323-335 (2011).

50. Nuñez-Lillo, G. et al. Identification of candidate genes associated with mealiness and maturity date in peach [Prunus persica (L.) Batsch] using QTL analysis and deep sequencing. Tree Genet. Genomes 11, 86 (2015).

51. Migicovsky, Z. et al. Genome to phenome mapping in apple using historical data. Plant Genome. 9, plantgenome2015.11.0113 (2016).

52. Dirlewanger, E. et al. Comparison of the genetic determinism of two key phenological traits, flowering and maturity dates, in three Prunus species: peach, apricot, and sweet cherry. Heredity 109, 280-292 (2012).

53. Lammerts, W. E. The breeding of ornamental edible peaches for mild climates, 1: inheritance of tree and flower characters-I. Inheritance of tree and flower characters. Am. J. Bot. 32, 53-61 (1945).

54. Dirlewanger, E., Graziano, E., Joobeur, T., Garriga-Calderé, F. \& Cosson, P. Comparative mapping and marker-Howad, W. and Ar $\mathrm{u}$ assisted selection in Rosaceae fruit crops. Proc. Natl Acad. Sci. USA 101, 9891-9896 (2004).

55. Beckman, T. G., Chaparro, J. X. \& Sherman, W. B. Evidence for control of double flowering in peach via dominant single gene loci. Acta Hortic. 962, 139-141 (2012).

56. Pascal, T. et al. Mapping of new resistance $(\mathrm{V} 2, \mathrm{Rm} 1)$ and ornamental (Di2, pl) Mendelian trait loci in peach. Euphytica 213, 132 (2017).

57. Stefano G. et al. Deletion of the miR172 target site in a TOE-type gene is a strong candidate variant for dominant double-flower trait in Rosaceae. Plant J. 96, 358-371 (2018)

58. François, L. et al. A miR172 target-deficient AP2-like gene correlates with the double flower phenotype in roses. Sci. Rep. 8, 12912 (2018).

59. Tang, $M$. et al. miR172 regulates both vegetative and reproductive development in the perennial woody plant Jatropha curcas. Plant Cell Physiol. 59, 2549-2563 (2018).

60. Zhu, Q. H., Upadhyaya, N. M., Gubler, F. \& Helliwell, C. A. Over-expression of miR172 causes loss of spikelet determinacy and floral organ abnormalities in rice (Oryza sativa). BMC Plant Biol. 9, 149 (2009).

61. Martínez-García, P. J. et al. High density SNP mapping and QTL analysis for fruit quality characteristics in peach (Prunus persica L.). Tree Genet. Genomes $\mathbf{9}$ 19-36 (2013).

62. Chakraborty, M. et al. Hidden genetic variation shapes the structure of functional elements in Drosophila. Nat. Genet. 50, 20-25 (2018).

63. Chin, C.-S. et al. Phased diploid genome assembly with single-molecule realtime sequencing. Nat. Methods 13, 1050-1054 (2016).

64. Chin, C.-S. et al. Nonhybrid, finished microbial genome assemblies from longread SMRT sequencing data. Nat. Methods 10, 563-569 (2013).

65. Walker, B. J. et al. Pilon: an integrated tool for comprehensive microbial variant detection and genome assembly improvement. PLoS One 9, e112963 (2014).
66. Langmead, B. \& Salzberg, S. L. Fast gapped-read alignment with Bowtie 2. Nat. Methods 9, 357-359 (2012).

67. Burton, J. N. et al. Chromosome-scale scaffolding of de novo genome assemblies based on chromatin interactions. Nat. Biotechnol. 31, 1119-1125 (2013).

68. Roberts, A., Pimentel, H., Trapnell, C. \& Pachter, L. Identification of novel transcripts in annotated genomes using RNA-Seq. Bioinformatics 27, 2325-2329 (2011).

69. Stanke, M., Steinkamp, R., Waack, S. \& Morgenstern, B. AUGUSTUS: a web server for gene finding in eukaryotes. Nucleic Acids Res. 32, W309-W312 (2004).

70. Haas, B. J. et al. Automated eukaryotic gene structure annotation using EVidenceModeler and the Program to Assemble Spliced Alignments. Genome Biol. 9, 1 (2008)

71. Chen N. Using Repeat Masker to identify repetitive elements in genomic sequences. Curr. Protoc. Bioinform. 5, 4.10.1-4.10.14 (2004).

72. Jurka, J. et al. Repbase update, a database of eukaryotic repetitive elements. Cytogenetic Genome Res. 110, 462-467 (2005).

73. Edgar, R. C. MUSCLE: a multiple sequence alignment method with reduced time and space complexity. BMC Bioinform. 5, 113 (2004).

74. Guindon, S. et al. New algorithms and methods to estimate maximumlikelihood phylogenies: assessing the performance of PhyML 3.0. Syst. Biol. 59 , 307-321 (2010).

75. Yang, Z. PAML 4: phylogenetic analysis by maximum likelihood. Mol. Biol. Evol. 24, 1586-1591 (2007)

76. Wang, L. R. \& Zhu, G. R. Descripters and Data Standard for Peach (China Agriculture Press, 2005).

77. Wang, L. R. Zhu, G. R., Fang W. C. Peach Genetic Resources in China (China Agriculture Press, 2012).

78. Li, H. Aligning sequence reads, clone sequences and assembly contigs with BWA-MEM. arXiv preprint at https:/arxiv.org/abs/1303.3997 (2013).

79. Li, H. Minimap2: pairwise alignment for nucleotide sequences. Bioinformatics 1 7-3100 (2018).

80. Li, H. et al. The sequence alignment/map format and SAMtools. Bioinformatics 25, 2078-2079 (2009).

81. McKenna, A. et al. The Genome Analysis Toolkit: a MapReduce framework for analyzing next-generation DNA sequencing data. Genome Res. 20, 1297-1303 (2010).

82. Purcell, S. et al. PLINK: a tool set for whole-genome association and population-based linkage analyses. Am. J. Hum. Genet. 81, 559-575 (2007).

83. Felsenstein, J. PHYLIP-phylogeny inference package (version 3.2). Cladistics 5, 163-166 (1989).

84. Alexander, D. H., Novembre, J., \& Lange, K. Fast model-based estimation of ancestry in unrelated individuals. Genome Res. 19, 1655-64 (2009).

85. Price, A. L. et al. Principal components analysis corrects for stratification in genome-wide association studies. Nat. Genet. 38, 904-909 (2006).

86. Zhang, C. et al. PopLDdecay: a fast and effective tool for linkage disequilibrium decay analysis based on variant call format files. Bioinformatics 35, 1786-1788 (2019).

87. Kang, H. M. et al. Variance component model to account for sample structure in genome-wide association studies. Nat. Genet. 42, 348-354 (2010).

88. Phanstiel, D. H., Boyle, A. P., Araya, C. L. \& Snyder, M. P. Sushi. R: flexible, quantitative, and integrative genomic visualizations for publication-quality multi-panel figures. Bioinformatics 30, 2808-2810 (2014).

89. Turner, S. D. qqman: an R package for visualizing GWAS results using QQ and Manhattan plots. Biorxiv 005165 (2014).

90. Kim, D., Langmead, B. \& Salzberg, S. L. HISAT: a fast spliced aligner with low memory requirements. Nat. Methods 12, 357-360 (2015).

91. Robinson, J. T. et al. Integrative genomics viewer. Nat. Biotechnol. 29, 24-26 (2011).

92. Pertea, M. et al. StringTie enables improved reconstruction of a transcriptome from RNA-seq reads. Nat. Biotechnol. 33, 290-295 (2015).

93. Livak, K. J. \& Schmittgen, T. D. Analysis of relative gene expression data using real-time quantitative PCR and the 2- $\triangle \triangle C T$ method. Methods 25, 402-408 (2001). 